\title{
Microstructural changes and in-situ observation of localization in OFHC copper under dynamic loading
}

Laurence Bodelot $^{\mathrm{a}, \mathrm{c}}$, Juan-Pablo Escobedo-Diaz ${ }^{\mathrm{b}, \mathrm{d}}$, Carl P. Trujillo ${ }^{\mathrm{b}}$, Daniel T. Martinez ${ }^{\mathrm{b}}$, Ellen K. Cerreta $^{\mathrm{b}}$, George T. Gray III ${ }^{\mathrm{b}}$, Guruswami Ravichandran ${ }^{\mathrm{a}}$

${ }^{a}$ Graduate Aerospace Laboratories, California Institute of Technology, Pasadena, CA 91125, USA

${ }^{\mathrm{b}}$ MST-8, Los Alamos National Laboratory, Los Alamos, NM 87545, USA

Current affiliations:

${ }^{\text {c }}$ Laboratoire de Mécanique des Solides, Ecole Polytechnique, 91128 Palaiseau, France

${ }^{\mathrm{d}}$ UNSW Australia, Canberra, ACT 2610, Australia

Corresponding author:

Laurence Bodelot - laurence.bodelot@polytechnique.edu

Laboratoire de Mécanique des Solides, Ecole Polytechnique

Route de Saclay

91128 Palaiseau Cedex, France

+33169335746

\begin{abstract}
:
In this paper, we introduce an original experimental protocol that couples microstructural analyses before and after deformation to in-situ grain scale strain measurements in OFHC copper samples during dynamic loading. This analysis is conducted on a modified shear compression specimen (SCS) that exhibits localization within a flat gage section and hence lends itself to ultra-high-speed imaging of the localization evolution. We were thus able to study the influence of the microstructure on strain localization as well as the process of localization in OFHC copper submitted to high-strain rate loading at different rates. We found that in the case of these modified perforated SCS samples, the early stages of localization were geometry driven while grain size and strain-rate dependency only emerged later. For the two smallest grain sizes examined here, a stronger strain-rate dependency was observed. This led to a narrower and more elongated localization of the highest strains at the local scale and to a concurrent harder macroscopic response at higher strain rate. For the largest grain size examined here, the macroscopic response was nearly identical at both strain rates but largely softer than for the smaller grain sizes. This translated into a wider localization pattern in the local strain fields compensating for lower values of maximum local strain. Texture evolution was strongly grain-size-dependent as smaller grain-size samples showed very mild lattice rotation accompanied with dynamic recrystallization into smaller grains, while the large grain-size samples showed a marked texture formation accompanied with both grain growth and stress relaxation phenomena. The introduced experimental protocol can thus give access to data providing new insight into microstructural aspects of localization under dynamic loading; such data is additionally relevant for validating multiscale or crystal plasticity models for dynamic applications.
\end{abstract}

Keywords: A. dynamics; B. polycrystalline material; C. electron microscopy; C. Kolsky bar; ultrahigh-speed imaging 


\section{Introduction}

Plastic localization has been largely studied experimentally in-situ at the microstructural scale under quasi-static loading (Efstathiou et al., 2010; Tschopp et al., 2009; Zhao et al., 2008). In the case of dynamic loading, the fact that the whole event takes place in under a few hundredths of milliseconds renders in-situ studies of plastic localization difficult to conduct, especially at a local scale. Though this work simply concerns plastic localization under dominant shear loading, most experimental studies at high strain rate have addressed the particular case of adiabatic shear banding or shear localization, in which an instability leads to the concentration of plastic deformation into a narrow band from an initial homogeneous state of shear (Zener and Hollomon, 1944). Note that such studies are mainly referred to in what follows with regard to their experimental approach. Whether their goal was to understand the influence of microstructure on localization (Cerreta et al., 2009, 2013; Dougherty et al., 2007) or to relate microstructural features to macroscopic mechanical quantities (Li et al., 2010; Meyers et al., 2003; Xue and Gray, 2006), the common trait of all these investigations is that they were limited to post-loading samples since none of the very few sample geometries that promote localization during high-strain-rate experiments are suited for in-situ diagnostics. For the top-hat specimen, used in numerous studies (Bronkhorst et al., 2006; Cerreta et al., 2009, 2013; Dougherty et al., 2007; Meyers et al., 2003; Xue and Gray, 2006), shear localization occurs within the volume of the specimen without emerging at the surface. For the thick-walled cylinder (Nesterenko et al., 1998), the external explosive technique used on the sample also prevents access to any in-situ observations of the microstructural evolution. This explains why researchers, who still attempted to study the timeline of localization, had to perform microstructural analyses on different specimens submitted to an increasing level of strain (Bai et al., 1994; Xue and Gray, 2006). They also had to evaluate the strain level from microstructural features. However, these features might have been — for the intermediate stages_affected by a cooling of the specimen and other relaxation processes. Thus these interrupted, post-loading testing techniques may not be an accurate snapshot of the strain and microstructure evolution during loading. Therefore, it is of great interest to perform in-situ measurements during high-strain-rate localization, as attested by the pioneering studies of Marchand and Duffy (1988) and Duffy and Chi (1992), who conducted initial work to examine just this with high-speed photography at multiple locations on a thin-walled cylinder submitted to dynamic torsion. Nevertheless, the magnification reached in their work limited the analysis to a rather mesoscopic scale and it is still a challenge today to access strain fields at the microstructural length scales believed to be important to determining the evolution of damage during high-strain-rate localization (Meyers et al., 1995). The development of ultra-highspeed imaging techniques now offers an opportunity to achieve this goal since it has recently permitted the measurement of in-situ strain fields under dynamic loading with a resolution in the order of the millimeter (Pierron and Forquin, 2012; Pierron et al., 2011).

Beyond the far-reaching question of the evolution of microstructure during localization, the large body of post-mortem work has helped to develop directed questions for in-situ study. For example, it was shown that higher strain rates promote narrower localization within adiabatic shear bands (Dougherty et al., 2009), but for a given material and strain rate, one wonders what parameters determine the size of the plastic localization features. In particular, the question remains as to what role is played by initial microstructural characteristics such as grain size. Based on the previous remarks, here we study the influence of grain size and strain rate on dynamic localization by conducting tests on samples of the same material having different grain sizes. For each sample, the microstructure of the area that experiences localization is characterized before and after loading, which will additionally give information regarding texture evolution. Furthermore, we aim at gaining a better understanding of the timeline and evolution of plastic localization by performing in-situ, full-field strain measurements. Such diagnostics require the use of a specimen for which localization occurs in a pre-determined and observable location. For that purpose, we will resort to a modified version of the shear compression specimen (SCS) and will also develop an original experimental setup to access in-situ, ultra-high-speed, strain measurements at the microstructural scale of an OFHC copper SCS. This study offers perspectives in providing novel experimental data regarding the influence of the microstructure on plastic localization under dynamic loading as well 
as a benchmark for the validation of recent multiscale models dedicated to both plasticity (Gao and Zhang, 2012; Khan et al., 2012) and localization (Baig et al., 2013; Sun et al., 2009; Zeng et al., 2015) under high-strain-rate loading.

The discussion of this work is arranged accordingly: (1) In the next section, the samples and preparation they underwent, as well as the experimental setup developed to access in-situ ultrahigh-speed strain measurements at the microstructural scale are described, (2) In Section 3, the results obtained from the different diagnostic tools employed in the study are discussed; and (3) Finally, the findings regarding the influence of the microstructure on plastic localization under dynamic shear loading are summarized.

\section{Materials and methods}

\subsection{Samples}

\subsubsection{Material}

The material studied in this paper is a 99.999\% pure OFHC (Oxygen Free High Conductivity) copper. After machining of the samples, the as-received material was heat-treated at different temperatures and dwell-times so as to provide samples with three different grain sizes (twin boundaries not included) as determined in a previous study (Escobedo et al., 2011); namely, $600^{\circ} \mathrm{C}$ for $1 \mathrm{~h}(60 \mu \mathrm{m}), 800^{\circ} \mathrm{C}$ for $1 \mathrm{~h}(100 \mu \mathrm{m})$, and $900^{\circ} \mathrm{C}$ for $35 \mathrm{~min}(200 \mu \mathrm{m})$. Since one of the goals of the study is to isolate the effect of grain size on localization, this particular material was selected because heat-treatment on OFHC copper is known to lead to grain growth without a significant effect on texture (Escobedo et al., 2011). This was further confirmed by analyzing the orientation data collected on each of the heat-treated samples (see Section 3.1).

\subsubsection{Specimen geometry}

While many sample geometries have been devised to induce shear localization during highstrain-rate experiments; many of these do not enable both in-situ observation of localization processes and/or post-mortem interrogation of microstructure. For example, the thick-walled specimen has two drawbacks: (1) it does not permit the generation of shear bands at desired locations, and (2) the external explosive technique associated with this sample also prevents in-situ visualization. A second example, the top-hat specimen, does lead to shear localization at a known location (namely, the junction between the brim and the hat), but this location is in the bulk of the sample. No modification of these samples, to our knowledge, could make in-situ observations of any type of localization possible.

However, there exists a sample that, with some adjustments described below, can overcome this issue; this sample is the shear compression specimen (SCS) developed by Rittel, Lee and Ravichandran (Rittel et al., 2002a, 2002b) and further characterized by Dorogoy and Rittel (2005a, 2005b). As described in Rittel et al. (2006), it consists of a cylindrical or parallelepiped body bearing two opposite grooves at $45^{\circ}$ with respect to the longitudinal axis (see Figure 1 ). The zone between the grooves is commonly referred to as the gage section and the flat surfaces at the bottom of these grooves are commonly referred to as the gage areas. The SCS is designed to generate very high-strain rates (of the order of $10^{4} \mathrm{~s}^{-1}$ ) in the gage section during loading in a split Hopkinson pressure bar (SHPB) setup and it has been shown in the aforementioned references that the geometry yields a homogeneous, dominant, shear state of stress in the gage section. Nevertheless, due to the abrupt change of geometry between the parallelepiped body and the grooves, stress concentrations occur on the sides of the gage section and ultimately lead to failure in the corners without further localization in the gage area. Unfortunately, this prevents any in-situ monitoring of the localization process. Note here that the primary purpose for the design of the SCS was to obtain 
very large shear strains and strain rates (Rittel et al., 2002a). As this task was largely completed before failure, the location of the failure was then irrelevant in the design.

In this study, two modifications of the initial SCS geometry are introduced: a root corner on both sides of the gage area (see arrows in Figure 1) for each opposite side of the gage section and a $0.5 \mathrm{~mm}$ diameter hole through the center of the gage section. The objective of these modifications is to promote localization around the hole at the center of the flat gage section and hence at a known and predictable location that is additionally accessible to in-situ ultra-high-speed imaging. While choosing the smallest hole that could be easily drilled, the root corner radius was adjusted thanks to Finite Element (FE) analysis with commercial software Abaqus. This enables the development of larger stress concentrations around the hole than along the sides of the gage area. For a hole of diameter $s=0.5 \mathrm{~mm}$, the selected root corner radius measures $R=0.6 \mathrm{~mm}$. Furthermore, systematic localization around the hole has been confirmed experimentally (Vaucorbeil et al., 2009). Pieces of differently heat-treated OFHC copper were machined into respective SCS specimens by Electric Discharge Machining (EDM) with the transverse direction of the as-received plate along the gage area of the sample. The dimensions of the sample are the following (see also Figure 1): $L=20 \mathrm{~mm}$, $W=10 \mathrm{~mm}, t=2.5 \mathrm{~mm}, w=3.70 \mathrm{~mm}, h=5.23 \mathrm{~mm}, R=0.6 \mathrm{~mm}, s=0.5 \mathrm{~mm}$ and $\theta=45^{\circ}$.

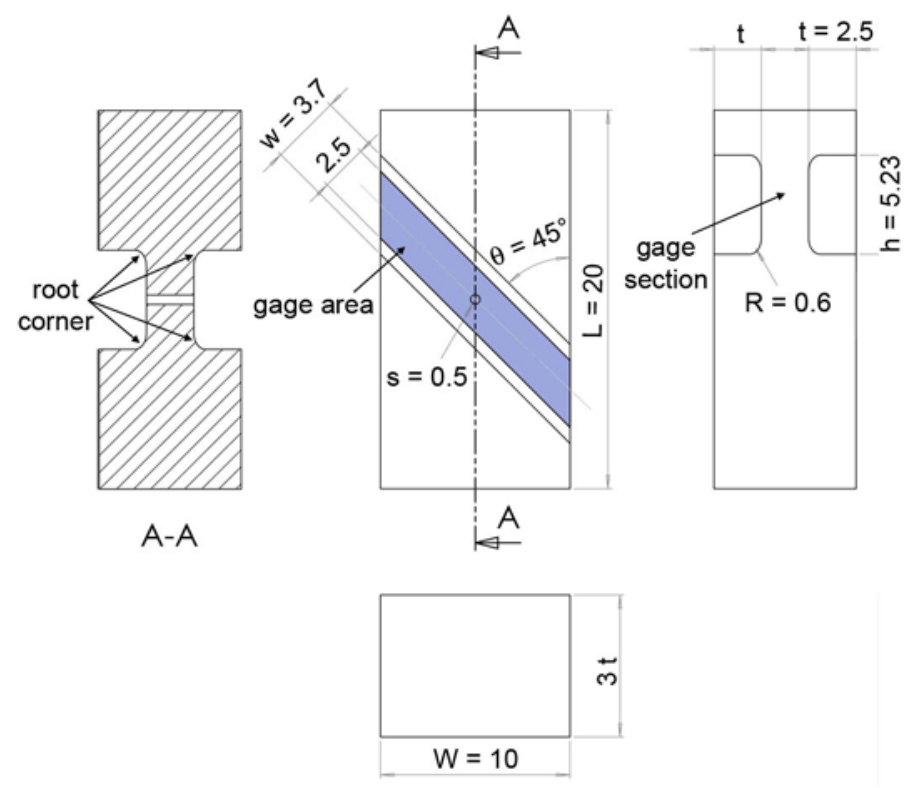

Figure 1. Geometry and dimensions (in millimeters) of the Modified Shear Compression Specimen (SCS) used in this study.

\subsubsection{Specimen preparation}

Prior to testing, the gage area of the SCS was polished to a mirror finish to allow for metallographic analysis of the specimen. Due to the narrow access to the gage section, this task was carried out manually, first with grinding papers of grades P400, P800, P1500 and P2500; then with diamond paste of grade $9 \mu \mathrm{m}, 3 \mu \mathrm{m}, 1 \mu \mathrm{m}$ and $0.25 \mu \mathrm{m}$ spread on a polishing cloth. Each diamondpolishing step was followed by chemical etching in a solution containing $40 \mathrm{~mL}$ of $\mathrm{H}_{2} \mathrm{O}, 5 \mathrm{~g}$ of $\mathrm{FeCl}_{3}$ and $12 \mathrm{~mL}$ of $\mathrm{HCl}$ and repeated twice. A last polishing step was carried with a colloidal silica solution of grade $0.05 \mu \mathrm{m}$. Finally the samples were electropolished in a solution containing $720 \mathrm{~mL}$ of $\mathrm{H}_{2} \mathrm{O}$ and $280 \mathrm{~mL}$ of $\mathrm{H}_{3} \mathrm{PO}_{4}$ at $2.1 \mathrm{~V}$ for 10 seconds.

Two samples for each of the three grain sizes studied in the paper were prepared to be tested at two different strain rates. These specimens are denoted $1 \mathrm{~L}, 1 \mathrm{H}, 2 \mathrm{~L}, 2 \mathrm{H}, 3 \mathrm{~L}, 3 \mathrm{H}$ where the number stands for the increasing grain size and the letters $\mathrm{L}$ and $\mathrm{H}$ stand for the lower strain rate and the higher strain rate, respectively. The strain rates achieved in the studied specimens are detailed in Table 2 within Section 3.2.

For post-loading EBSD measurements, the specimens' outer bodies were ground to allow for the preparation of the gage section. Once the gage section was reached, the surface was prepared using 
only P4000 grinding paper to limit the amount of material removal. The samples were then polished with $1 \mu \mathrm{m}$ and $0.05 \mu \mathrm{m}$ colloidal silica solutions, followed by electro-polishing using the solution as before.

\subsection{Microstructural characterization}

After metallographic preparation, and before the test, crystallographic orientations within the gage area of each sample were collected using a Philips XL 30 Field Emission Scanning Electron Microscope equipped with an EBSD module. The electron gun was operated at $20 \mathrm{kV}$ and at a spot size of 4. Because of the particular shape of the SCS, conducting EBSD measurements over the gage area, which lies at the bottom of the groove, was not straightforward. To permit such orientation collection, the gage section needed to be carefully positioned in the SEM chamber so that the electron beam could reach and reflect from the surface with minimal hitting of the borders of the gage section while the sample was tilted at $70^{\circ}$. For the SCS geometry used in this study, with a groove height $(w)$ of $3.7 \mathrm{~mm}$, only a $2.5 \mathrm{~mm}$-wide zone could thus be scanned without any reflection issues.

Optical images of the deformed samples were acquired post-mortem thanks to a Zeiss Imager M2 Axio microscope. Orientation data collection was then performed again after mechanical testing and sample preparation.

\subsection{High-strain-rate testing}

High-strain-rate experiments were conducted in a SHPB setup. Tests on OFHC copper SCS required the use of high-modulus bars with a diameter larger than the width $W$ of the samples. Hence, both the incident and transmitted bars were made of 350 maraging steel and were $19.05 \mathrm{~mm}$ in diameter and 1,226.2 mm in length. The incident and reflected pulses, as well as the transmitted pulse, were collected by strain gages located in the middle of the incident and transmitted bars, respectively. The incident bar was struck by a $19.05 \mathrm{~mm}$-diameter maraging steel striker propelled by a gas gun. The duration and amplitude of the pulse were controlled by the striker length and the release pressure of the gas gun. In this study, two different strain rates were achieved through utilization of a $203.2 \mathrm{~mm}$-long striker launched at $19 \mathrm{psi}$ for the lower strain rate $\left(\sim 4,400 \mathrm{~s}^{-1}\right.$ on average), and a $165.1 \mathrm{~mm}$-long striker launched at $21 \mathrm{psi}$ for the higher strain rate $\left(\sim 5,100 \mathrm{~s}^{-1}\right.$ on average). A $16.5 \mathrm{~mm}$-long tempered maraging steel hollow cylinder, in which a window was cut out for in-situ observation of the sample, was placed around the SCS during the experiment. The purpose of this sleeve was to absorb aftershocks since once the SCS had been loaded, the bars would hit the sleeve. It also prevented the sample from undergoing additional deformation while it was falling out of the bars. This ensured that the post-mortem state of the sample actually matched the final state of strain measured in-situ.

Displacement and stress-strain curves in cylindrical samples loaded within a SHPB setup are commonly derived directly from the data collected by the above-mentioned strain gages via the well-known relationships detailed by Kolsky (1949). However, in the case of the SCS, obtaining the stress-strain relationship in the gage area of the sample requires additional processing. Firstly, since a SCS with a rectangular section is used to permit a homogeneous lighting of the gage area (see Section 2.4.), the interface between the bars and the sample is more prone to wave dispersion. Hence, the SHPB data is corrected for dispersion (following the derivation by Bancroft (1941)) before further processing. Subsequently, the data reduction procedure for the SCS, described by Dorogoy and Rittel (2005a, 2005b) can be carried out. It leads to the following formulas giving the Von Mises equivalent stress and equivalent plastic strain in the gage area:

$$
\sigma_{e q}=k_{1}\left(1-k_{2} \varepsilon_{e q}^{p}\right) \frac{P}{W t} \text { and } \varepsilon_{e q}^{p}=k_{3} \frac{d}{h},
$$


where $P$ is the applied load, $d$ is the prescribed displacement applied at the specimen interface, $W$ is the specimen width, $t$ is the gage thickness and $h$ is the gage height. $k_{1}, k_{2}$ and $k_{3}$ are material- and geometry-dependent coefficients that are to be determined by FE analysis (Dorogoy and Rittel, 2005a, 2005b), as described in the next paragraph. Note that though the formula were initially introduced in a study using a circular section SCS, they also apply to a SCS with a rectangular section: it was indeed shown in Alkhader and Bodelot (2012) that, after data reduction using Equation (1), equivalent stress-strain curves obtained from rectangular section SCSs were in good agreement with stress-strain curves obtained from cylindrical samples.

In the FE analysis, only half of the SCS needs to be modeled due to symmetry considerations. The mesh is refined in the gage section and even more in the root corner radii to account for the need for precision and the strong section variations in these locations, respectively (see Figure 3a); this leads to a mesh containing 720,614 elements in total and 410,805 elements across the width of the groove (root corners included). While the surface at the bottom is fixed, the top surface is submitted to a downward displacement. The material behavior used as input (true stress-strain curve) in the model comes from experimental compression tests performed close to the strain rate range of interest on cylindrical samples of OFHC copper that underwent the heat treatments described in Section 2.1. These curves are reported in Figure 2 and are nearly identical for all heattreated specimen, exhibiting an inflection point around $8 \%$ strain before further hardening. The FE simulation then gives the following outputs: average force $P$ on the top surface and average displacement $d$ of the top surface, as well as the average equivalent stress $\sigma_{e q}$ in the gage area midsection and the average equivalent plastic strain $\varepsilon^{p}{ }_{e q}$ in the gage area mid-section. Finally, the $k_{\mathrm{i}}$ parameters are obtained by performing a least-squares fit of the two following functions:

$$
\frac{\sigma_{e q} W t}{P}=f\left(\varepsilon_{e q}^{p}\right)=k_{1}-k_{1} k_{2} \varepsilon_{e q}^{p} \text { and } \varepsilon_{e q}^{p}=f\left(\frac{d}{h}\right)=k_{3} \frac{d}{h} \text {. }
$$

This analysis was performed using the commercial software Abaqus (a plot of the equivalent Von Mises stress in the gage area under $1 \mathrm{~mm}$ prescribed displacement is shown in Figure $3 \mathrm{~b}$ ). The coefficients obtained for the SCS geometry described in Figure 1 and the material behaviors linked to the three different grain sizes are reported in Table 1.

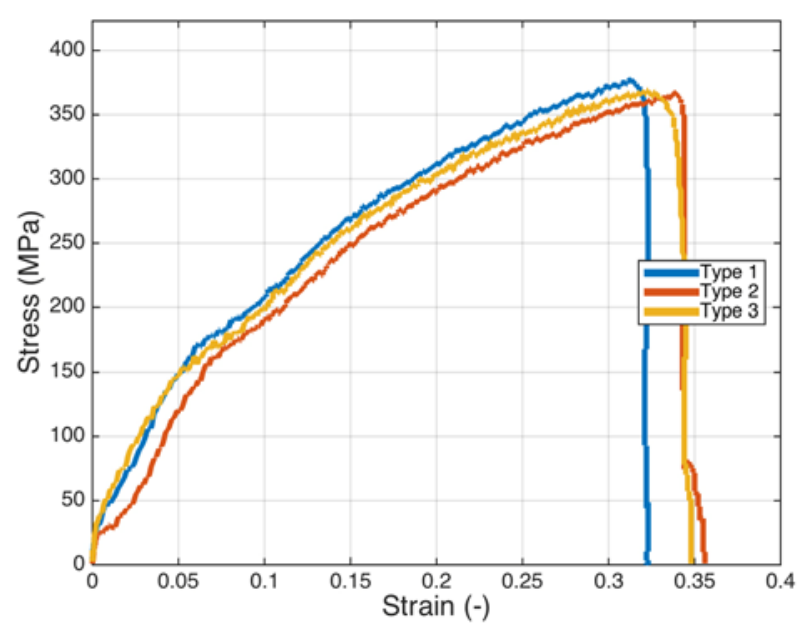

Figure 2. Stress-strain curves obtained through SHPB testing at $\sim 3,000 \mathrm{~s}^{-1}$ on cylindrical samples of OFHC copper that underwent the same heat treatments than the SCS samples. 


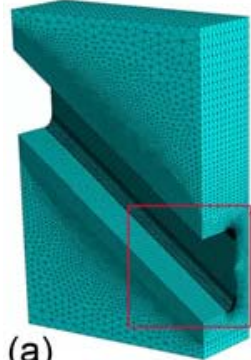

(a)

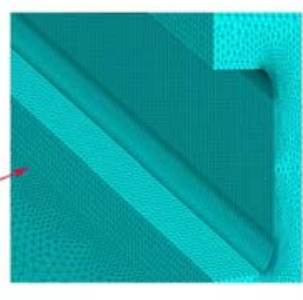

.

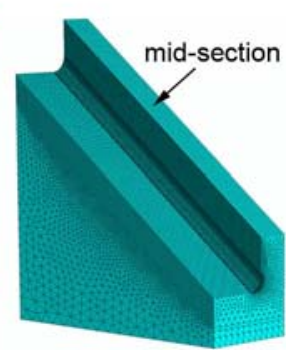

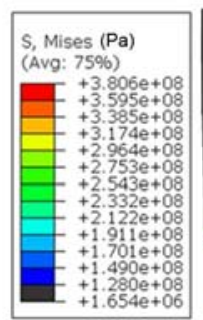

(b)

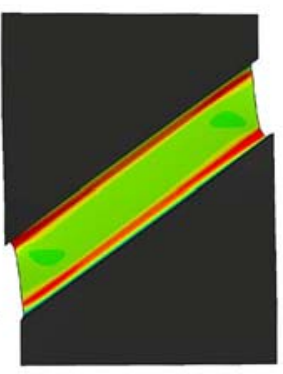

Figure 3. (a) Views of the mesh of a half SCS modeled in Abaqus to obtain the coefficients used in the data reduction procedure giving the stress-strain relationship in the SCS gage area from the SHPB loading parameters. (b) FEM results showing the equivalent Von Mises stress in the gage area for a $1 \mathrm{~mm}$ prescribed displacement of the top surface.

\begin{tabular}{|c|c|c|c|}
\hline Sample heat treatment & $k_{1}$ & $k_{2}$ & $k_{3}$ \\
\hline $600^{\circ} \mathrm{C}-1 \mathrm{~h}$ & 0.9174 & 0.1188 & 0.5836 \\
\hline $800^{\circ} \mathrm{C}-1 \mathrm{~h}$ & 0.9292 & 0.1426 & 0.5838 \\
\hline $900^{\circ} \mathrm{C}-35 \mathrm{~min}$ & 0.9192 & 0.1798 & 0.6028 \\
\hline
\end{tabular}

Table 1. For the three different grain sizes considered in this study, coefficients $k_{1}, k_{2}$ and $k_{3}$ used in the data reduction procedure giving the stress-strain relationship in the SCS gage area from the SHPB loading parameters.

\subsection{In-situ ultra-high-speed imaging}

Images of the deforming sample were taken with a HPV-2 Shimadzu camera. This camera has a full matrix of 312 x 260 pixels and is made of an In-situ Storage Image Sensor (Kondo et al., 2003), more commonly called ISIS-CCD sensor, capable of recording a maximum of a hundred 10-bit images at one million frames per second. In this work, the frame rate was set to half-a-million frames per second with an exposure time of a quarter interframe to obtain sharp, full-frame images during the entire loading process.

The HPV-2 camera was equipped with an Infinity K2 long-distance microscope mounted with a TR tube and a CF3 lens, and was placed in front of the SHPB setup, where the sample was held between the incident and the transmitted bars, so that the flat gage area of the SCS, which was coated with a speckled paint, was in focus. A BK7 sheet of glass, transparent to visible wavelength, was placed at the end of the Infinity lens to protect it if the sample and sleeve were to be ejected from the apparatus (see Figure 4). The resulting field of view, for a working distance of $101 \mathrm{~mm}$, was $4.74 \mathrm{~mm} \times 3.95 \mathrm{~mm}$. This covered the smallest surface of gage area needed for observing localization around the hole while reaching the best resolution possible, namely $15.2 \mu \mathrm{m} /$ pixel.

With ultra-high-speed imaging, the lighting, specifically intensity, stability and timing, of the sample is crucial. In these experiments, two Photogenic Power Light 2500DR flash lamps were positioned on both sides of the camera, so as to get homogeneous lighting in the SCS gage area at full intensity (see Figure 4). Synchronization of the SHPB apparatus, lighting and ultra-high-speed camera worked as follows: just before hitting the incident bar, the striker cut through a laser beam which served as an immediate trigger to the lamp that required $100 \mu$ s to reach full illuminating intensity and stayed on for $400 \mu \mathrm{s}$; this also triggered the recording of the gages signals, as well as the camera with a $200 \mu$ s delay. This synchronization enabled imaging during the loading of the sample while the light intensity was steady. In this way, images were generated that were readily correlatable to the data recorded by the strain gages.

Strain fields were finally obtained by processing the images of the deforming sample with Digital Image Correlation (DIC). Pursuant to the requirements of this technique (Chu et al., 1985), a random speckle pattern covering a wide range of gray levels was applied onto the gage section of the SCS prior to testing. The gage area was thus finely coated with white acrylic paint before being 
speckled with black acrylic paint; both paints were applied with an airbrush to obtain a very fine speckle pattern (see Figure 5). To allow for DIC analysis, a reference image is collected and divided into square subsets, all having a unique signature of gray levels due to the speckle; these subsets can subsequently be tracked in the following images thus leading to displacement fields and strain fields. More details about this technique can be found in a recent comprehensive overview book by Sutton et al. (2009).

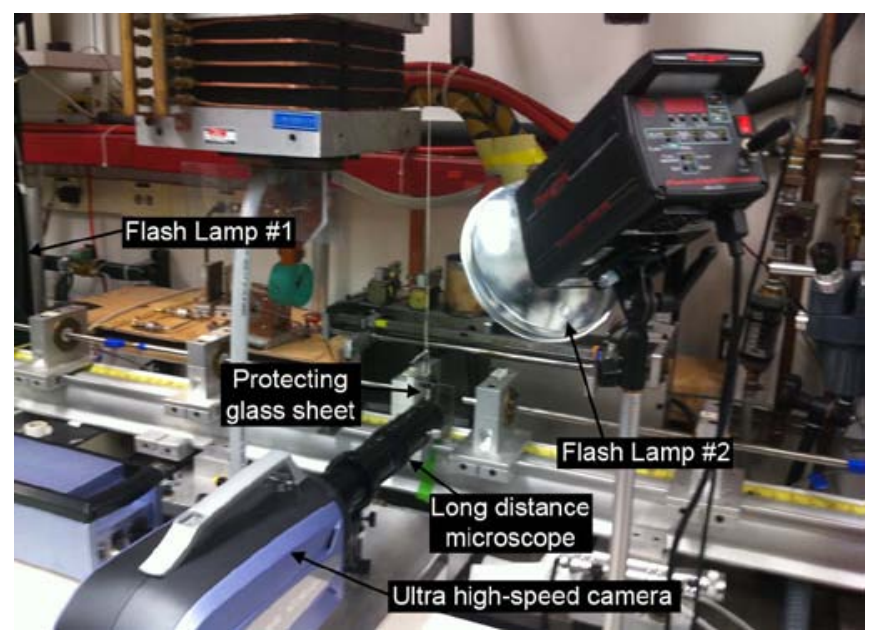

Figure 4. Experimental setup for dynamic, in-situ, strain measurements in the gage area of a SCS loaded within a SHPB apparatus. Note, one of the two flash lamps is out of the field of view.

In this work, data was processed in incremental mode (reference image is updated as the previous image at each correlation step) by the VIC-2D software from Correlated Solutions Inc. A high-order interpolation scheme (8-tap spline) was selected for the conversion of digital data into continuous data. The correlation criterion chosen to analyze the image was the Zero-Normalized Sum of Squared Differences (ZNSSD), which is particularly insensitive to noise and light fluctuations (Sutton et al., 2009). Considering that large strains are expected during high-strain-rate plastic localization, the strains computed from the displacement fields are finite strains and they are expressed in the reference configuration (Green-Lagrange strain tensor). Note that, because the outof-plane strain cannot be captured, the Von Mises strain is computed with the plane strain formula and will be referred to as "in-plane Von Mises strain" in what follows. Finally, the subset size, a compromise between accuracy and resolution, was taken as $13 \times 13$ pixels with a 3 pixel step size, which corresponds to subsets of $197.6 \mu \mathrm{m}$ x $197.6 \mu \mathrm{m}$ with a step of $45.6 \mu \mathrm{m}$. The relative sizes of the gage area, subset and speckle are reported in Figure 5.

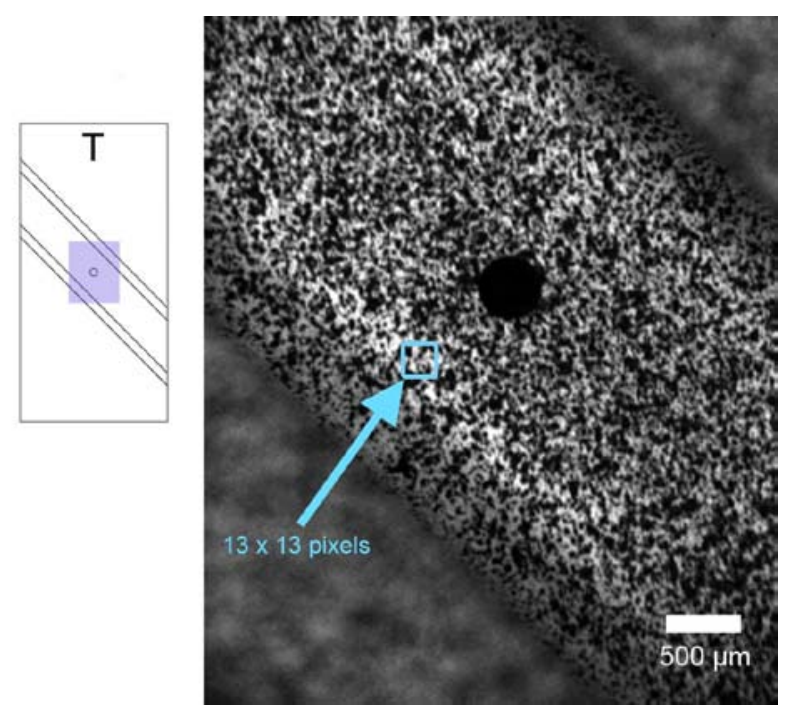

Figure 5. Zone of interest in sample 1L covered with a fine speckle and imaged by the Shimadzu camera and subset size used for DIC data processing. The left-hand-side inset is a schematic of the location of the zone imaged with respect to the sample. 


\section{Results and discussion}

\subsection{Initial microstructure and texture}

The orientation maps relative to the sample's normal direction obtained by the TexSEM Laboratory (TSL) processing software are reported for all samples in Figure 6, along with their corresponding pole figures. The location of the zone imaged within the sample, as well as the color scale of the orientation maps, are provided as insets at the left of Figure 6. The pole figures show that all the samples exhibit an essentially random texture. Additionally, a preliminary visual inspection of these pole figures seems to indicate that there is little variation in texture between all of the studied samples, as noticed in earlier works (Escobedo et al., 2011).

The data collected by EBSD was further analyzed to compute the orientation distribution (OD) of each sample in Rodrigues space. As detailed in Bodelot and Ravichandran (2014), working in Rodrigues space has the advantage of allowing for the evaluation of the statistical correlation between two discrete ODs via the Pearson's chi-square test (Pearson, 1900). The OD of sample 1H (smallest average grain size) was taken as the reference distribution and chi-squares values were computed by taking every other OD as the second dataset. The obtained values were then compared to the chi-square probability threshold at 95\%, which corresponds to the point of distinction between significance (underneath the threshold) and non-significance (above the threshold), in terms of statistical correlation, according to Wilson and Hilferty (1931). As shown in Figure 7, all chi-square values but one fall below the chi-square threshold, which means that the ODs of the samples that underwent further grain growth due to annealing prior to mechanical testing remain strongly correlated to the OD of the sample that underwent the least grain growth, except for sample $3 \mathrm{H}$ that slightly deviates from the initial texture. It is unlikely that this could be attributed to initial sample-to-sample variability as all samples used in this study were cut out from the same laminated plate but, since heat treatments were performed post-machining, variability in the heat treatment process cannot be excluded, especially at higher temperature and shorter dwell time, as it is the case for type 3 samples. Actually, sample $3 \mathrm{H}$ in Figure $6 \mathrm{f}$ exhibits a larger grain size distribution than sample 3L in Figure 6c, which indicates that more annealing took place in sample $3 \mathrm{H}$. Hence, despite some variability in sample $3 \mathrm{H}$, the different heat treatments performed on the samples did not lead to significantly different textures and texture variations can be ruled out as a parameter of influence in the localization processes considered in this study. However, since a slight deviation is observed already in sample $3 \mathrm{H}$, it seems that further grain growth would eventually lead to nonnegligible texture evolution. 

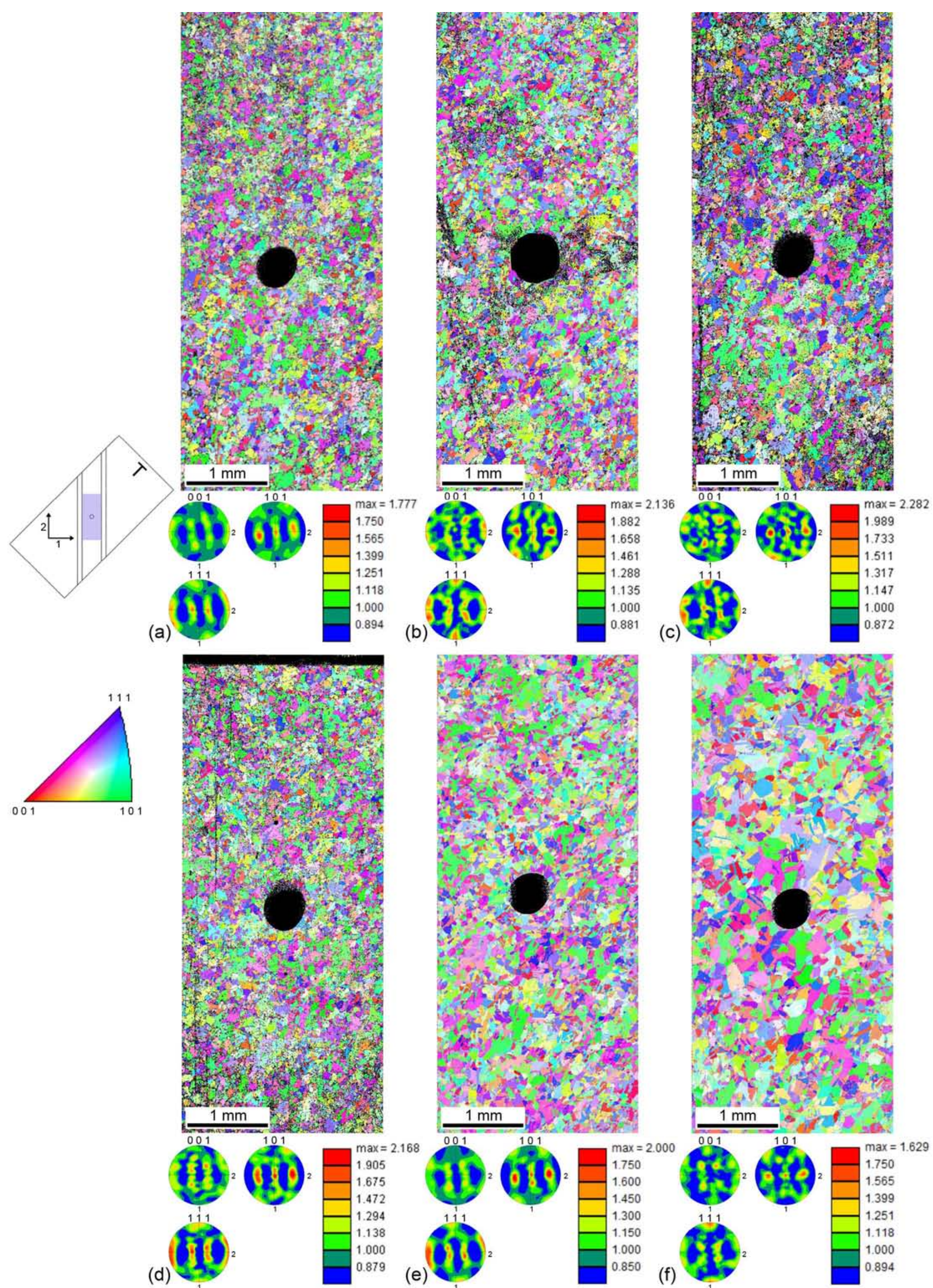

Figure 6. EBSD scans obtained before mechanical testing in the gage area of sample (a) 1L, (b) 2L, (c) 3L, (d) $1 \mathrm{H}$, (e) $2 \mathrm{H}$ and (f) $3 \mathrm{H}$. The left-hand-side insets show a schematic of the location of the imaged zone with respect to the sample and the color scale used for the orientation maps. 


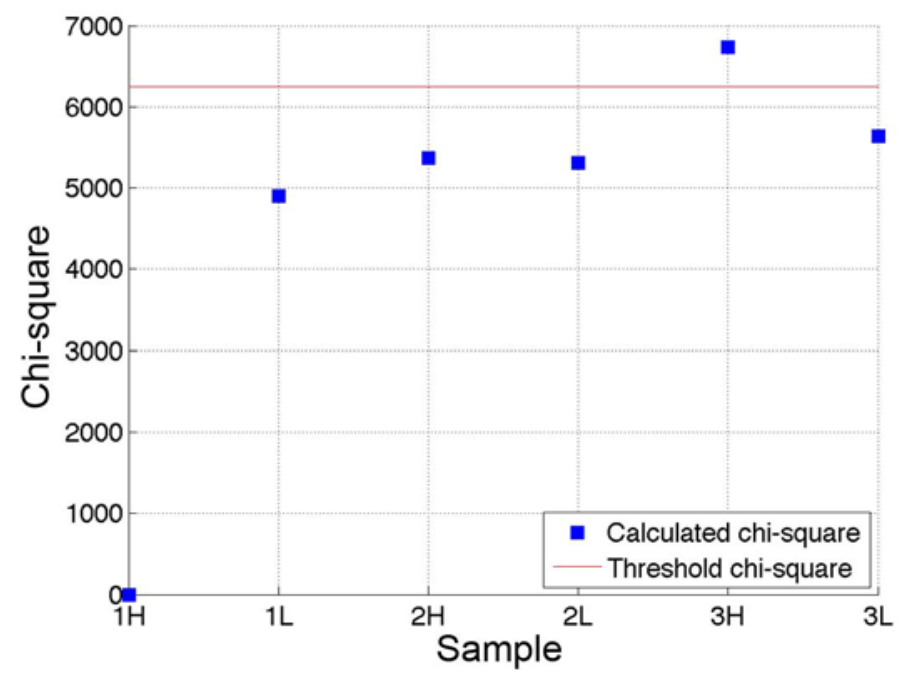

Figure 7. Chi-square test for statistical correlation in terms of orientation distribution between sample $1 \mathrm{H}$ and the other samples, showing that the ODs of samples that underwent further grain growth remain essentially correlated to the OD of the sample that underwent the least grain growth.

\subsection{Macroscopic high-strain rate behavior}

Signals from the strain gages of the SHPB apparatus were processed using the data reduction procedure described in Section 2.3. The equivalent strain rates achieved in each sample are reported in Figure 8a and summarized in Table 2. The lower, average, strain rates are $\sim 4,300 \mathrm{~s}^{-1}$ for both samples $1 \mathrm{~L}$ and $2 \mathrm{~L}$, and $\sim 4,500 \mathrm{~s}^{-1}$ for sample 3L. The higher average strain rates are $\sim 5,000 \mathrm{~s}^{-1}$ for samples $1 \mathrm{H}$ and $2 \mathrm{H}$, and $\sim 5,200 \mathrm{~s}^{-1}$ for sample $3 \mathrm{H}$. These curves suggest that type 1 and type 2 samples-whose grain sizes are rather close-have a very similar behavior while type 3 samplesthat have a distinctly larger grain size-behave differently from the other samples. The equivalent stress-strain curves are plotted for all samples in Figure 8b. Samples of equivalent grain size do not exhibit significant strain-rate sensitivity over this narrow strain rate regime, except above $17 \%$ strain for type 1 and 2 samples. Indeed, the stress-strain curves of samples $1 \mathrm{~L}, 2 \mathrm{~L}, 1 \mathrm{H}$ and $2 \mathrm{H}$ overlap (up to $17 \%$ ) while the curves of samples $3 \mathrm{~L}$ and $3 \mathrm{H}$ are relatively close to each other during the whole loading. Beyond $17 \%$ strain, the response of samples loaded at the lower strain rate is significantly softer than the response of samples loaded at the higher strain rate, the latter showing further hardening. Besides, the stress-strain curves for the samples of larger grain size (type 3 samples) show a non-negligible softer behavior throughout the loading, in accordance with the Hall-Petch effect.
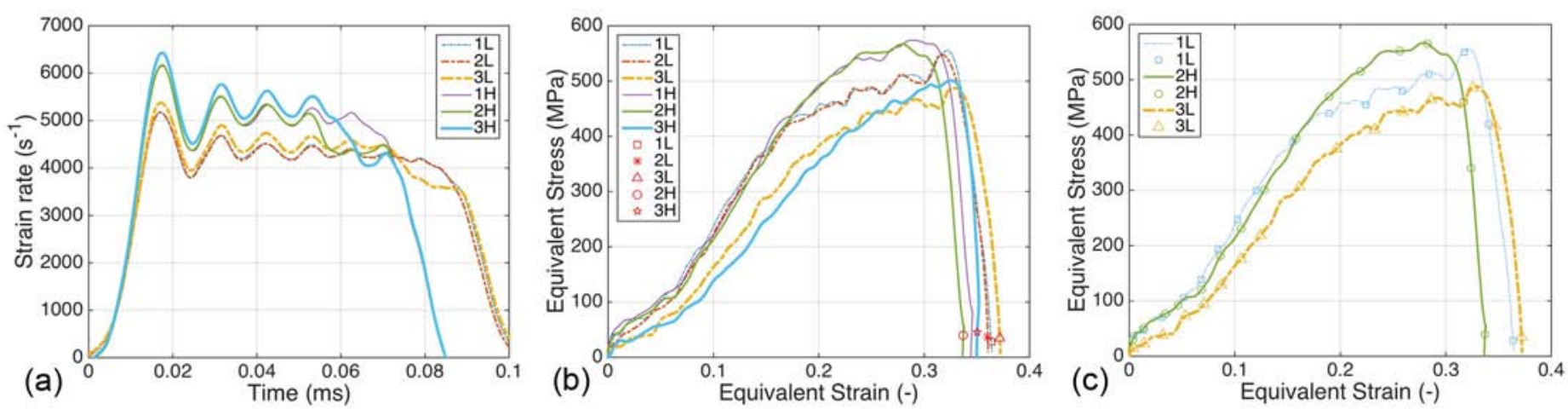

Figure 8. (a) Equivalent strain rates achieved in the tested samples. (b) Equivalent stress-strain curves of the tested samples. (c) Equivalent stress-strain curves for samples 1L, 2H and 3L with indication of the points for which the strain field timeline will be plotted. 


\begin{tabular}{|c|c|c|c|c|c|c|}
\hline Sample & $1 \mathrm{~L}$ & $1 \mathrm{H}$ & $2 \mathrm{~L}$ & $2 \mathrm{H}$ & $3 \mathrm{~L}$ & $3 \mathrm{H}$ \\
\hline Average grain size & $60 \mu \mathrm{m}$ & $60 \mu \mathrm{m}$ & $100 \mu \mathrm{m}$ & $100 \mu \mathrm{m}$ & $200 \mu \mathrm{m}$ & $200 \mu \mathrm{m}$ \\
\hline Average strain rate & $4,300 \mathrm{~s}^{-1}$ & $5,000 \mathrm{~s}^{-1}$ & $4,300 \mathrm{~s}^{-1}$ & $5,000 \mathrm{~s}^{-1}$ & $4,500 \mathrm{~s}^{-1}$ & $5,200 \mathrm{~s}^{-1}$ \\
\hline
\end{tabular}

Table 2. Summary of average grain size and testing strain rate for each studied sample.

\subsection{In-plane strain fields in the gage area and timeline of localization}

Since samples $1 \mathrm{~L} / 2 \mathrm{~L}$ and $1 \mathrm{H} / 2 \mathrm{H}$ exhibit the same macroscopic constitutive curves (see Section 3.2 and Figure $8 \mathrm{~b}$ ), only the fields obtained during loading on samples $1 \mathrm{~L}$ and $2 \mathrm{H}$ (out of the four above-mentioned ones) are presented for the sake of brevity. In the same way for the larger grain size, only the fields obtained on sample 3L are shown. Snapshots of the in-plane Von Mises strain fields obtained at the instants marked in Figure 8c are thus plotted in Figure 9 (respectively Figure 10 and Figure 11) and give the timeline of strain localization in sample 1L (respectively sample $2 \mathrm{H}$ and sample 3L). Note that the span of the scale is updated over the test duration to highlight the development of strain localization within the gage area without saturation of the color scheme. However, for each shown sample, fields marked a) and b), c) and d), e) to h), i) to l) and m) to p) are respectively plotted with identical scales to allow for comparison between samples. 


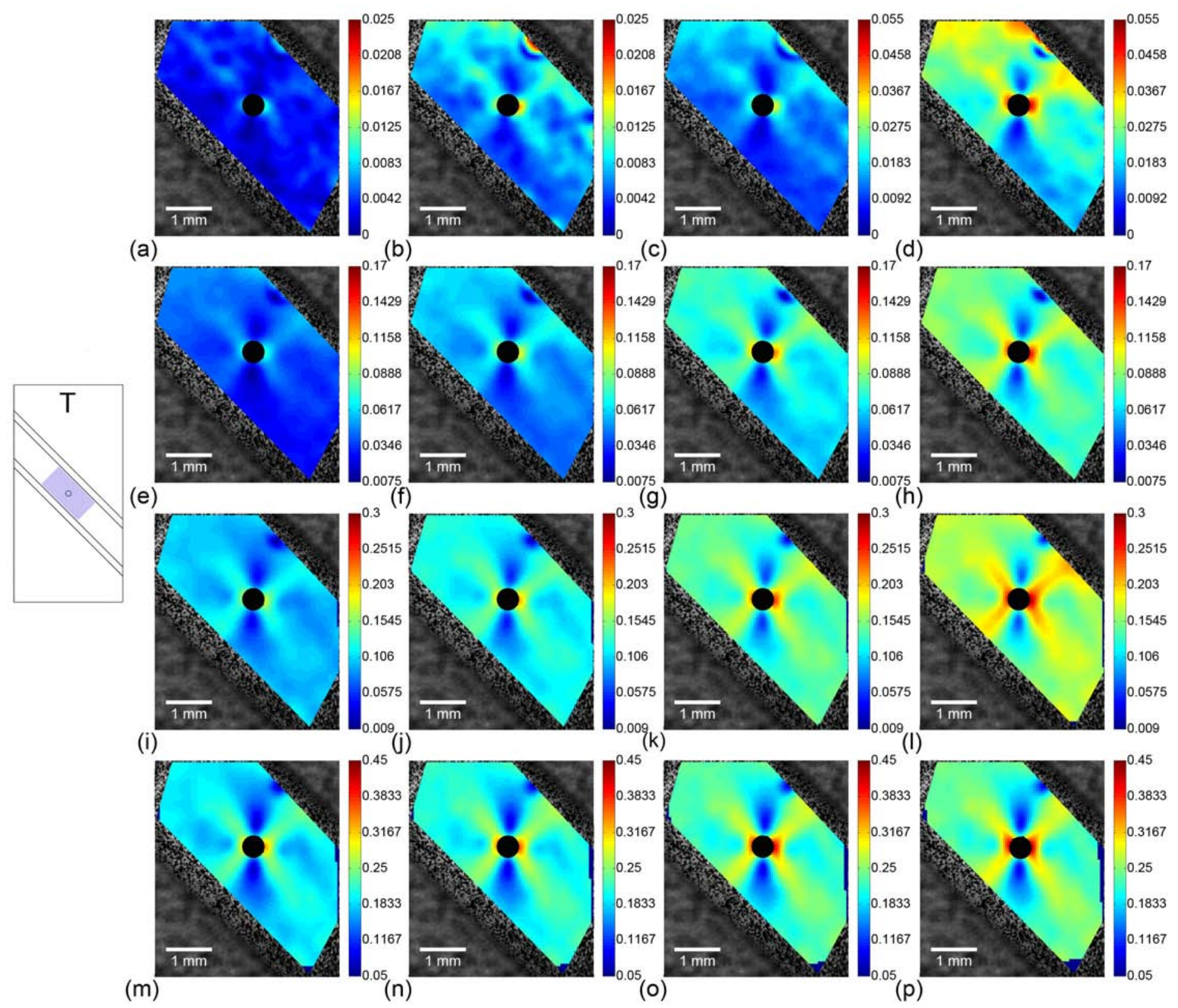

Figure 9. In-plane equivalent Von Mises strain fields obtained during loading of sample 1L at different instants reported in Figure 8c and plotted in the reference or undeformed configuration. The left-hand-side inset is a schematic of the location of the strain field computation zone with respect to the sample. 


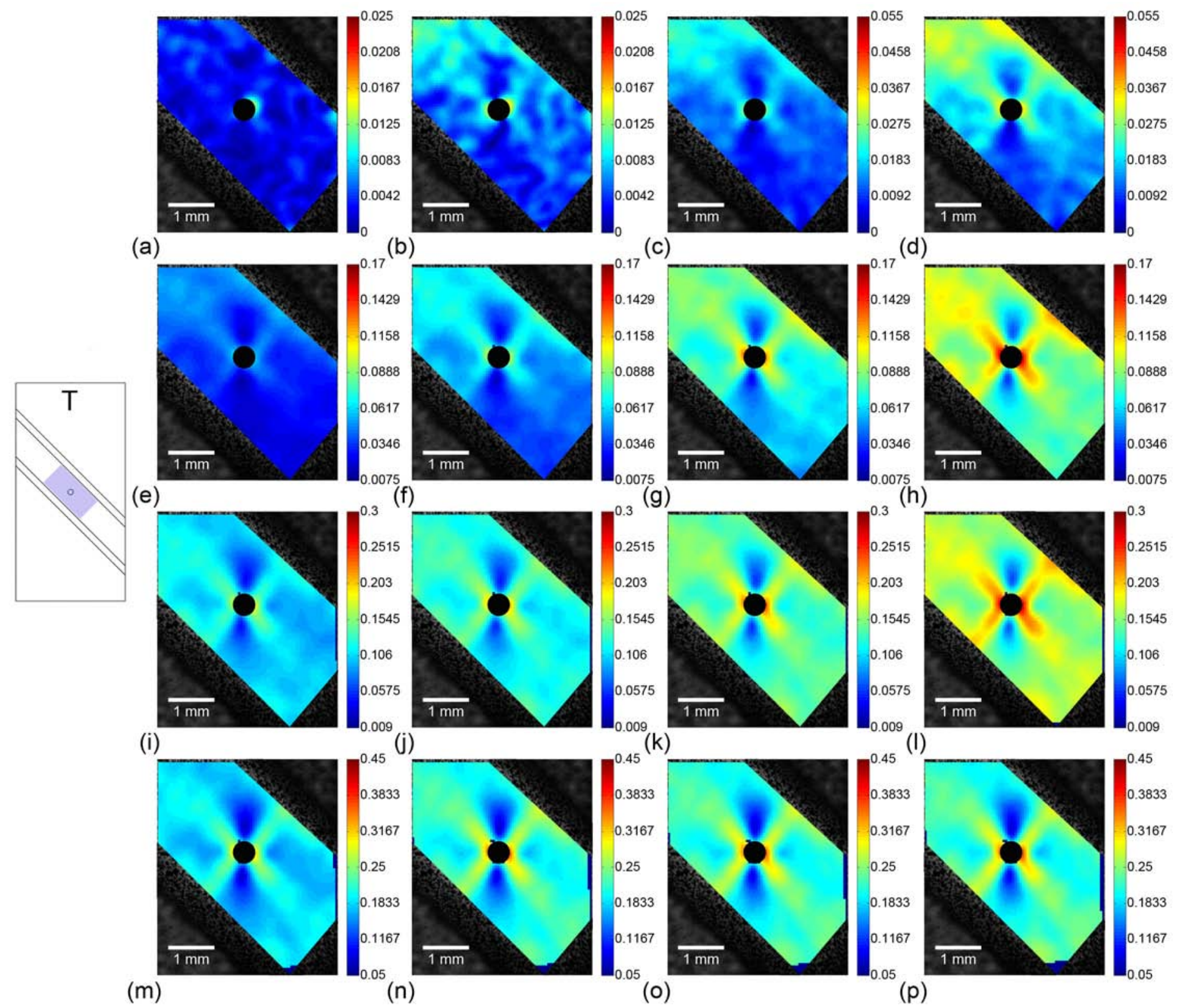

Figure 10. In-plane equivalent Von Mises strain fields obtained during loading of sample $2 \mathrm{H}$ at different instants reported in Figure $8 \mathrm{c}$ and plotted in the reference or undeformed configuration. The left-hand-side inset is a schematic of the location of the strain field computation zone with respect to the sample. 


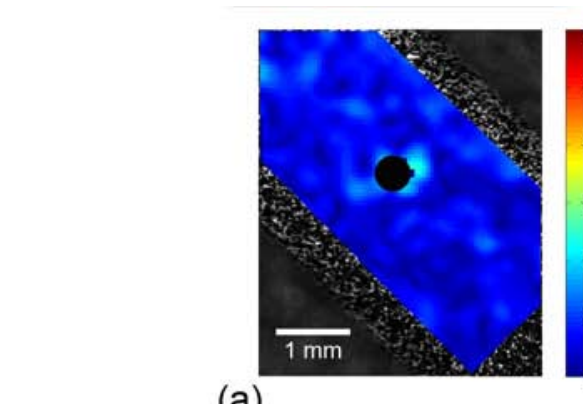

(a)

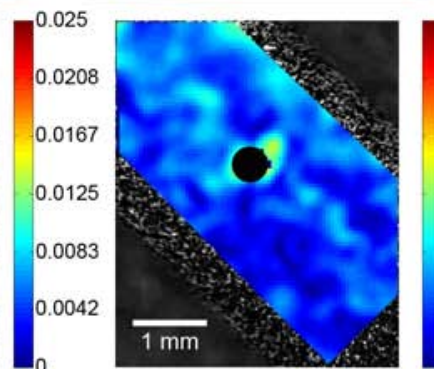

(b)

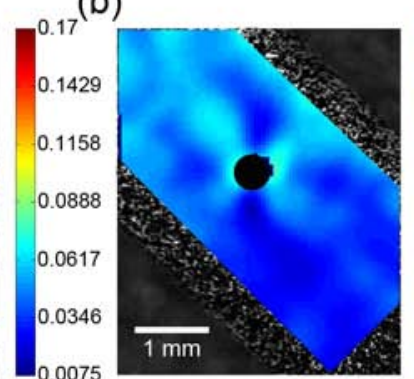

(e)
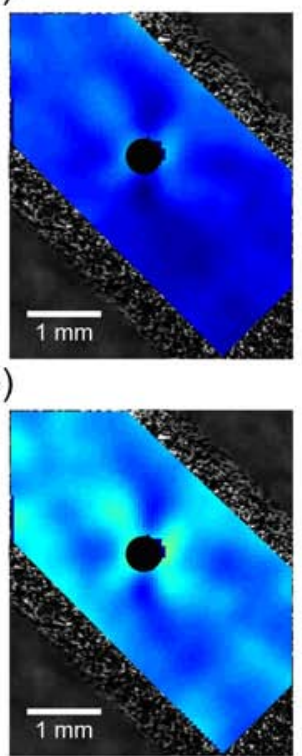

(i)

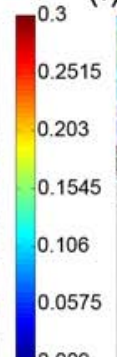

f)

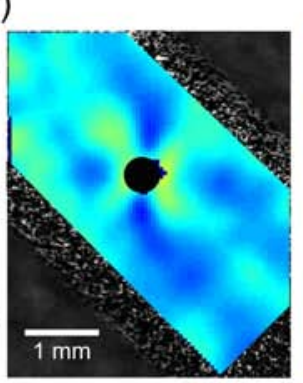

(j)

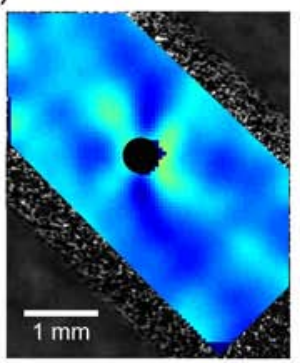

(m)

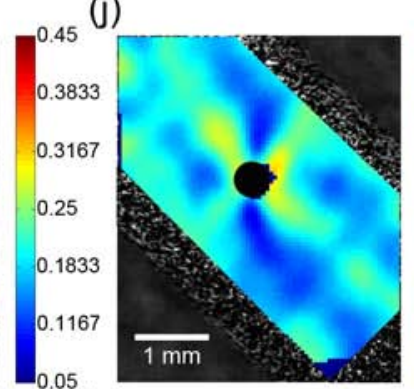

(n)

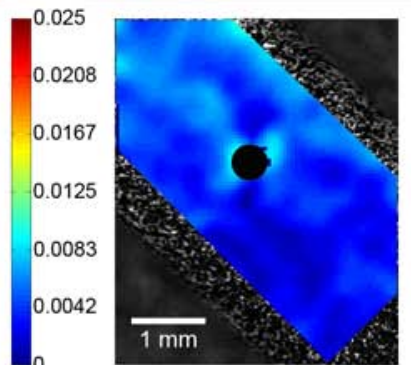

(c)

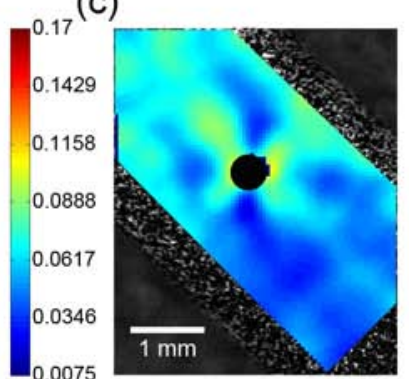

(g)

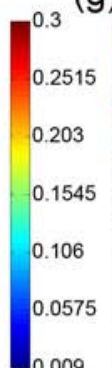

(k)

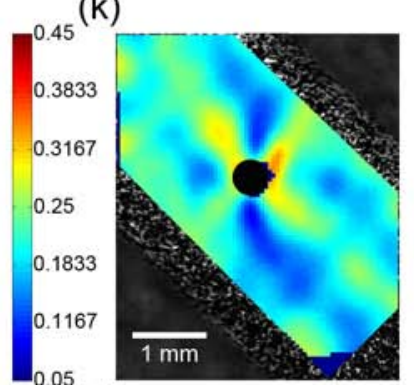

(o)

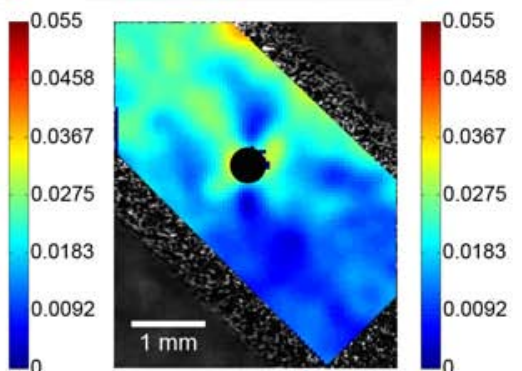

(d)

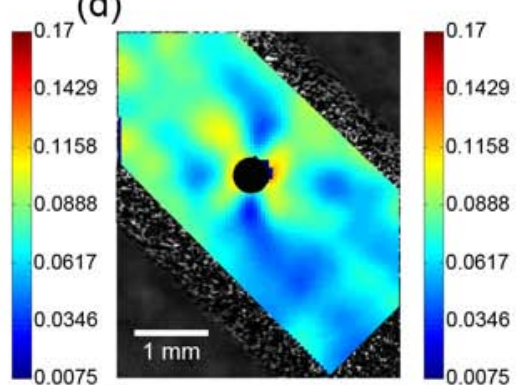

(h)

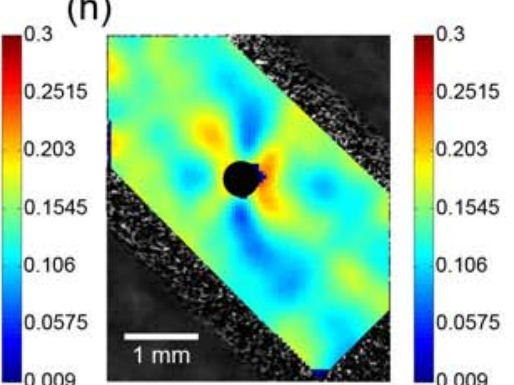

(I)

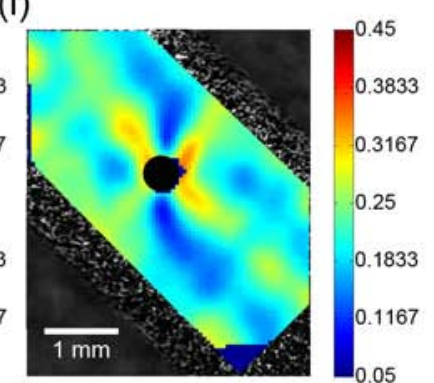

(p)

Figure 11. In-plane equivalent Von Mises strain fields obtained during loading of sample 3L at different instants reported in Figure 8c and plotted in the reference or undeformed configuration. The left-hand-side inset is a schematic of the location of the strain field computation zone with respect to the sample.

Before commenting on the fields in Figure 9 through Figure 11, it is important to mention that all the DIC computations were run with the same subset size; hence the smoothing due to the averaging of the strain within the subset is the same for all compared fields. Additionally, since the fields are plotted in the reference or undeformed configuration to allow for more comfortable analysis and comparison of their evolution during loading, the hole appears to remain circular throughout the plotted sequence of strain fields though it actually progressively closes (full closure happen for all samples), as will be seen in the post-loading observations of Section 3.4. In all considered samples, early plastic localization exhibits a "butterfly" shape around the hole, which is typical of perforated samples (Timoshenko and Woinowsky-Krieger, 1959) (see steps b and c in Figure 9 through Figure 11). The following strain fields show a progressive spreading and intensification of the butterfly-shaped localization zone within the gage area of the sample (up to step h in Figure 9 through Figure 11). This suggests that the early stages of localization, both in terms of shape and timeline, are geometry-driven since they are similar for all considered samples 
regardless of their microstructure or imposed strain rate for loading. Beyond that point, the localization zone retains its general butterfly shape and exhibits increasing strain values throughout the loading. Towards the end of the loading, one can notice that the zone of highest strains within the butterfly area tends to get narrower, but with a different spread depending on the considered sample. The final stages of the localization, and in particular the details of the final features of the localization zone, thus seem to be driven by the microstructure.

Figure 9 l-m-n and Figure 10 l-m-n also allow for a qualitative comparison of the local strains between samples having a similar grain size but loaded at different strain rate, namely samples $1 \mathrm{~L}$ and $2 \mathrm{H}$. At the macroscopic scale, the stress-strain curve of the latter exhibits more hardening because of a higher loading strain rate while at the microstructural scale, the area corresponding to the highest values of the in-plane Von Mises strains exhibits narrower fingers within the butterfly shape. This is consistent with the formula derived by Dodd and Bai (1985), in which the width of a shear band is inversely proportional to the strain rate.

To investigate, in more detail, the influence of the microstructure on the final features of the localization zone, the in-plane equivalent Von Mises strain fields upon unloading (see markers in Figure $8 b$ ) are plotted for all tested samples with their own full scale in Figure 12 so that the bounds of each scale correspond to the minimum and maximum strain values within the gage area. Note that strain data is missing for sample $1 \mathrm{H}$ due to a light bulb bursting during the experiment.

(a)

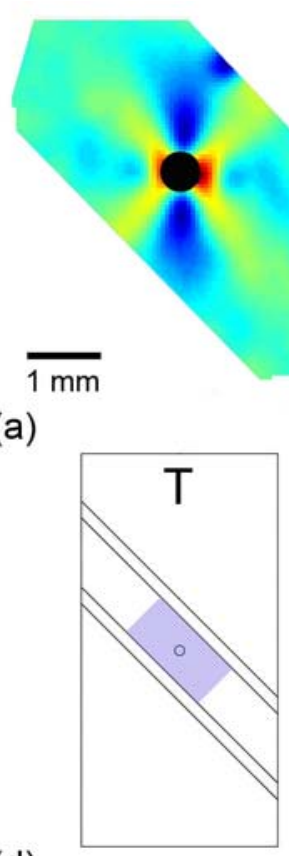

(d)

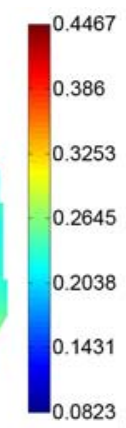

(b)
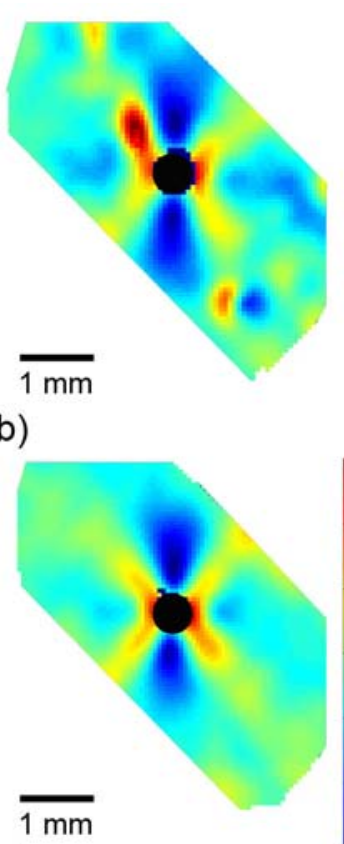

(e)

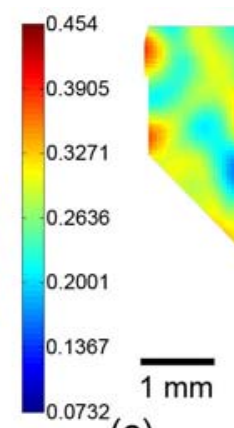

(c)

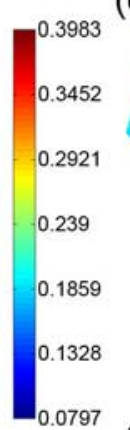

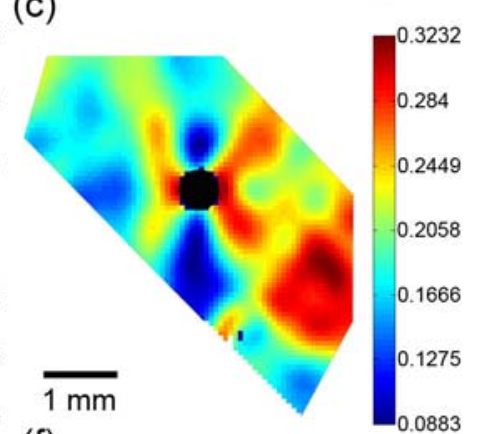

(f)

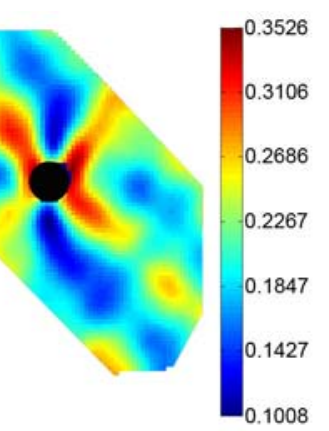
.

. 
of boundary length are less prone to dislocation pile-ups at boundaries due to enhanced development of dislocation tangles in the interior areas of the grain. Pile-ups are thought to be important sources for stress concentrations. Hence, less opportunity to form pile-ups leads to fewer opportunities for stress concentration development. Therefore the larger grained specimens are less prone to high strain values at grain boundaries in the localization area. These lower values of maximum local strain for larger grain sizes are actually compensated by a much wider area including high values of strain, finally leading, overall, to average higher macroscopic residual strains for large-grain samples as expected from the macroscopic curves.

In the macroscopic stress-strain curves in Figure 8b, samples $1 \mathrm{~L}, 2 \mathrm{~L}$, and to a much lesser extent $3 \mathrm{~L}$, also exhibited less hardening than samples $1 \mathrm{H}, 2 \mathrm{H}$ and $3 \mathrm{H}$, respectively, due to a lower rate of loading for the former. At equivalent grain sizes, the samples loaded at the lower strain rate could finally accommodate larger deformations. This translates at the microstructural scale to higher local residual strains and a notably larger localization area for the smaller grain-size samples. For the large grain-size samples, the most noticeable difference concerns the peak value of residual strains that is also higher for the sample loaded at the lower rate. From these observations regarding the final features of the local strain fields, it follows that the extent of the area corresponding to the highest strains is primarily driven by the grain size and correlates with it. For a given grain size, the strain rate of the loading drives mainly the maximum local value of the local strain, a higher strain rate leading to more hardening, hence lower values of the maximum local strain.

\subsection{Post-loading microstructure}

Post-mortem optical images of the gage areas are reported in Figure 13. These micrographs show that all samples exhibit hole closure. They also suggest qualitatively that there is an increase in final roughness that correlates with grain size while there is very little difference in roughness as a function of strain rate.

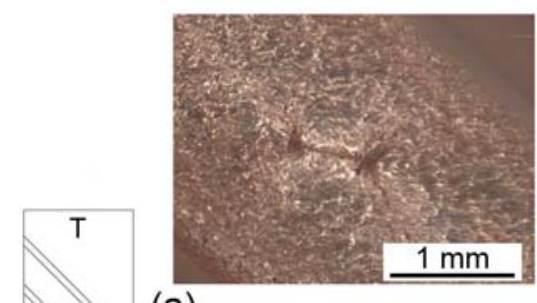

(a)

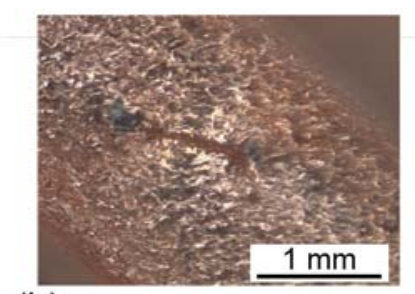

(b)
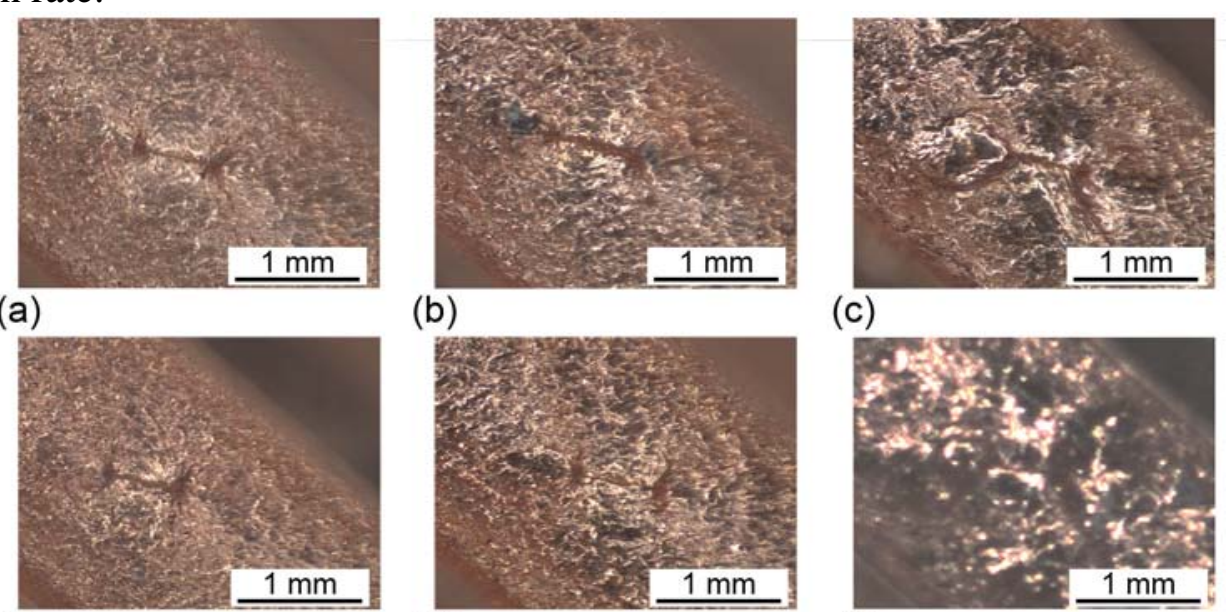

(d)

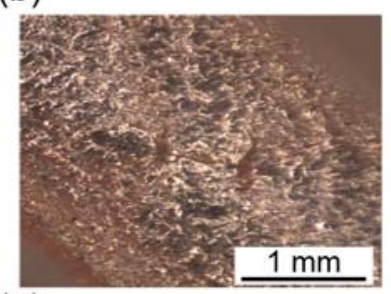

(e) (c)

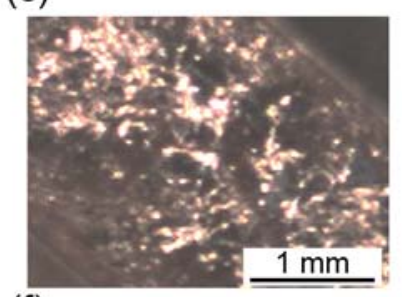

(f)

Figure 13. Micrographs at the center of the gage section (where the hole was) after high-strain-rate mechanical testing for sample (a) 1L, (b) $2 \mathrm{~L}$, (c) $3 \mathrm{~L}$, (d) $1 \mathrm{H}$, (e) $2 \mathrm{H}$ and (f) $3 \mathrm{H}$. The left-hand-side inset is a schematic of the location of the zone imaged with respect to the sample.

To study the changes undergone by the crystalline orientations induced by plastic localization, and more precisely the extent of lattice rotation, ESBD was performed in the gage area of all samples after high-strain-rate deformation. However, the deformation experienced in the gage section during mechanical testing has caused the groove height $w$ to diminish from $3.7 \mathrm{~mm}$ to $2.6 \mathrm{~mm}$ on average, thus narrowing down the width to be scanned. Post-loading EBSD scans relative to the sample's normal direction performed on all tested samples are reported in Figure 14. Note, the large dark areas in the scans of post-loading samples are artifacts due to electron charging at the slit location. 

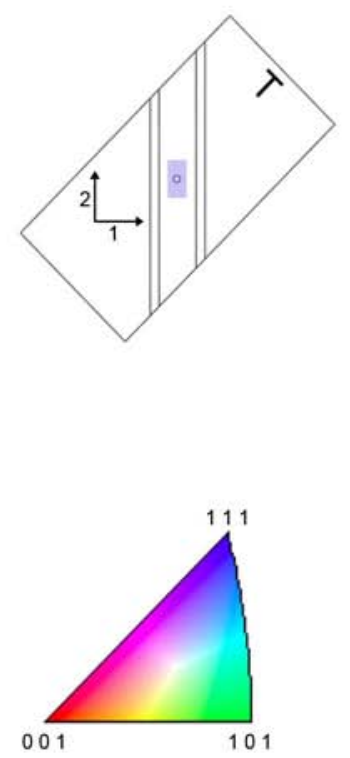

(a)
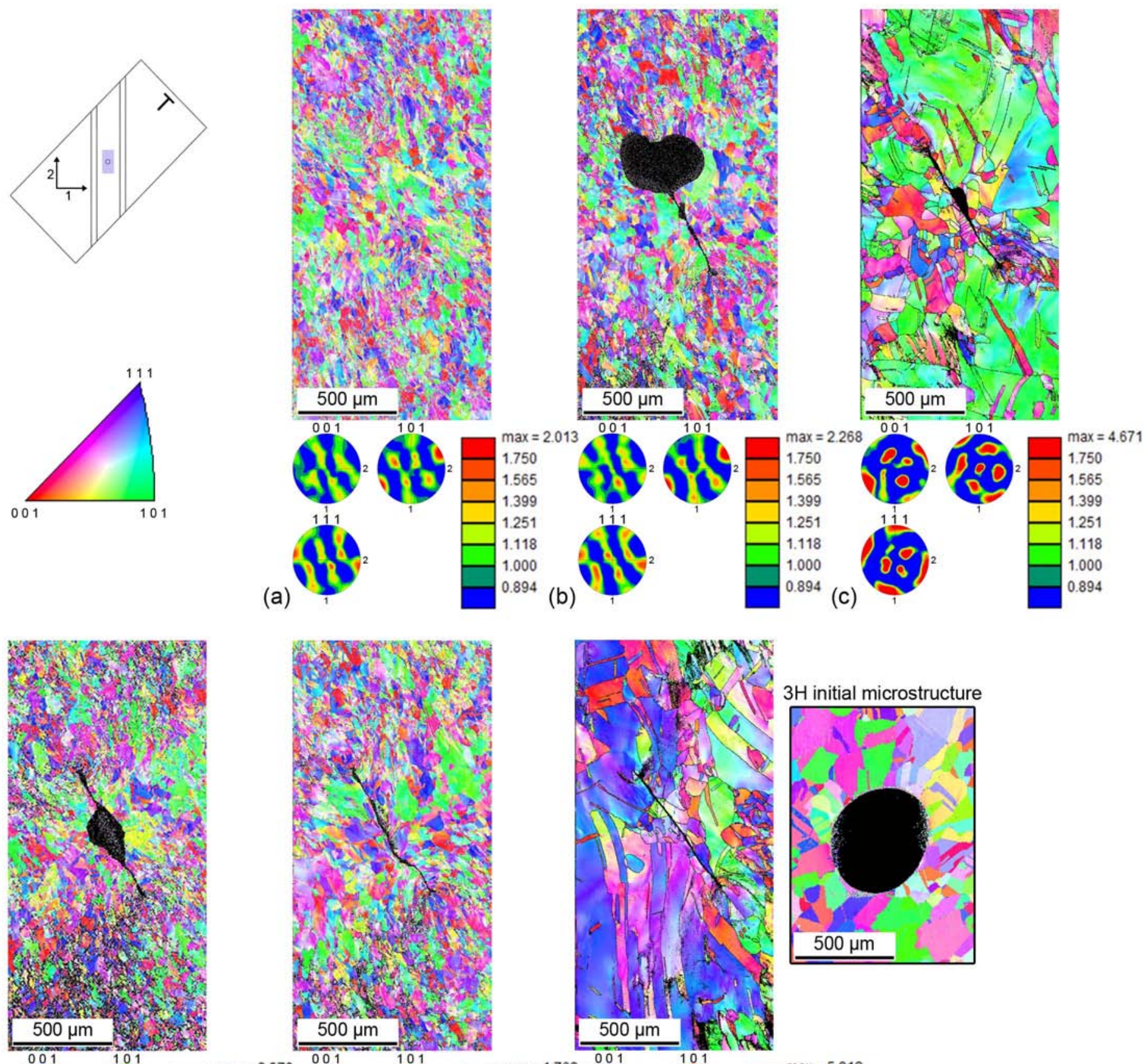

(b)

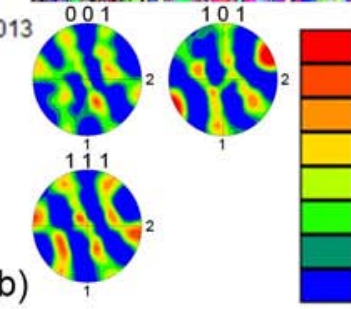

(c)
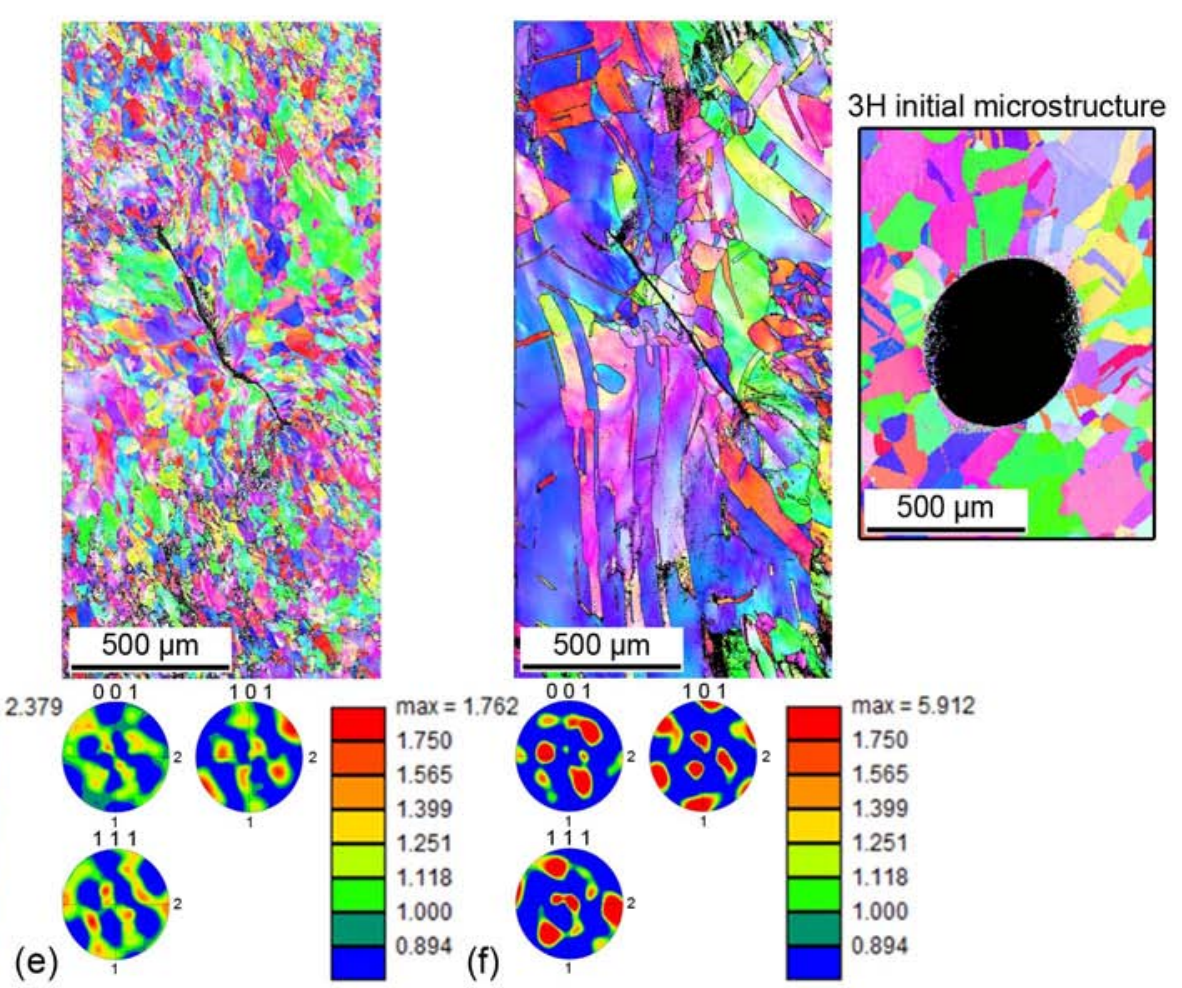

(d)
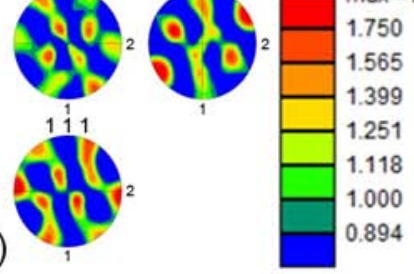

(e)

(f)

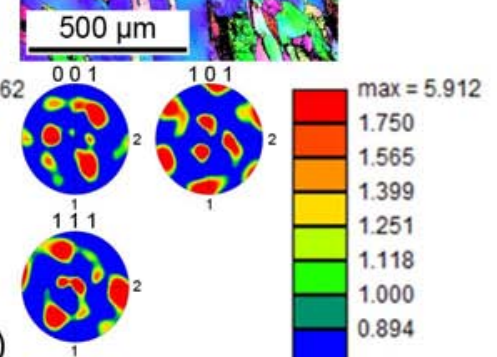

Figure 14. EBSD scans obtained after high-strain-rate mechanical testing in the gage area of sample (a) $1 \mathrm{~L}$, (b) $2 \mathrm{~L}$, (c) $3 \mathrm{~L}$, (d) $1 \mathrm{H}$, (e) $2 \mathrm{H}$ and (f) $3 \mathrm{H}$ with inset showing the initial microstructure. The top left-hand-side inset is a schematic of the location of the zone imaged with respect to the sample. Note the difference of scale, as well as the reduced area of data collection, between Figure 6 and Figure 14. Except for the inset, the large dark areas in the scans are artifacts due to electron charging at the slit location. 
By comparing the EBSD scans and their corresponding pole figures before and after loading (Figure 6 and Figure 14), one can observe that many grains in the deformed gage section display misorientation development (lattice rotation) due to shearing. This is most obviously tracked through grains displaying a high variation in colors indicating a high variation in orientations in a single grain, as well as through an evolution of the features of the pole figures. This lattice rotation took place in the gage area of all of the tested samples, however this phenomenom is mild for the smaller grained specimens while significantly pronounced for the larger grained specimens. For samples $1 \mathrm{~L} / 1 \mathrm{H}$ and $2 \mathrm{~L} / 2 \mathrm{H}$ (Figure $14 \mathrm{a}-\mathrm{b}-\mathrm{d}-\mathrm{e}$ ), the grains located in the localization area elongated along the localization pattern. Some grains also appear smaller than in the virgin sample, which testifies of an intense shearing but also suggests a localized dynamic recrystallization. The microstructural changes in samples 3L/3H (Figure $14 \mathrm{c}-\mathrm{f}$ ) with initially larger grains appear quite different. Grains elongated much more strongly along the localization pattern and the most striking feature in these samples is the presence of extended zones of identical orientation, which indicates firstly a marked texture formation. In addition, the grain boundaries were overlaid on the orientation maps as black lines in Figure 14c and in Figure 14f and a partial map of the virgin 3H sample was inserted to highlight the fact that some grains are now larger in the deformed large-grain samples, thus revealing a possible grain growth process or, at least, a clustering of rotations towards the same orientation. Though the phenomenon of dynamic recrystallization leading to smaller grains inside shear localization bands has largely been observed (Cerreta et al., 2009; Chen et al., 1999; Dougherty et al., 2009; Meyers et al., 2003), the above-mentioned phenomenon leading to these larger grains has never been reported before following high-strain-rate loading in copper. This might be explained by the fact that high-strain rate testing is rarely performed on initially largegrained copper since hardening is much more desirable than softening in impact applications. From a modeling perspective, to our knowledge, the only treatment of dynamic recrystallization leading to grain growth has been made by Wenk and Tomé (1999) after observation of the phenomenom in dynamically sheared olivine-a coarse-grained mineral. According to them, soft grains, because they accumulate more disclocation, are less stable and hence more likely to be consumed by less deformed grains through boundary migration. The influence of the temperature rise has however not been discussed and is, in our opinion, non negligible in the process.

Furthermore, as was discussed in the previous section dedicated to strain fields measurements, despite their higher macroscopic residual strains, samples with the largest grain size exhibit lower maximum local strains than samples with smaller grain sizes. The strain fields suggested that lower maximum strain values were compensated by the fact that areas of higher strains were more widespread in large-grain samples. The very large lattice rotations evidenced in the present section also suggest that a non negligible amount of the strain experienced within large grains may come from out-of-plane strains, which cannot be captured by the in-plane DIC measurements of local strain fields.

Finally, evidence of recrystallization in large-grain samples was further provided by maps of kernel average misorientation (KAM) computed from the EBSD scans and reported in Figure 15. The KAM within a grain is calculated by averaging the misorientation angles between each data point and all its nearest neighbors. It is proportionally correlated to the amount of accumulated strain in non recrystallized samples (Cerreta et al., 2013). In samples 1L, 1H, 2L and 2H (Figure 15a, d, b and e, respectively), the distributions of KAM values in the zone of interest correlate well with the corresponding final strain fields. One indeed note KAM values larger than one in nearly the whole gage area (except very sparingly where the in-plane Von Mises strains were minimum) while the highest values delineate the shape of the localization zone. On the contrary, samples 3L and $3 \mathrm{H}$ (Figure 15c and $\mathrm{f}$ ) only exhibit a few occurences of high KAM values in the localization area and mostly low or null values where the apparent large grains are located. Hence, KAM values indicate very little accumulated strains in these locations in spite of the large strains experienced. This is the evidence that recrystallization took place and lead to grain growth along with very intense stress relaxation. This process was very likely thermally-assisted. 


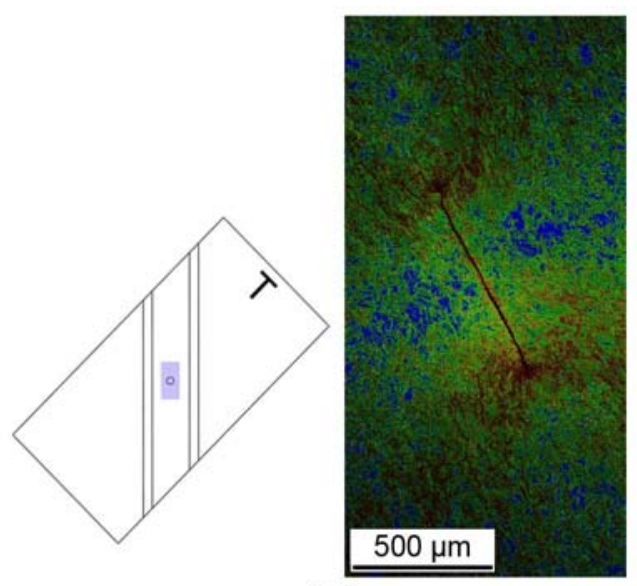

(a)

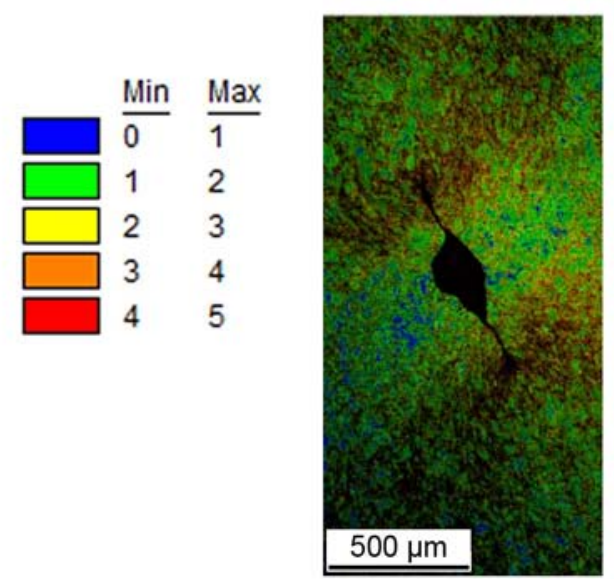

(d)

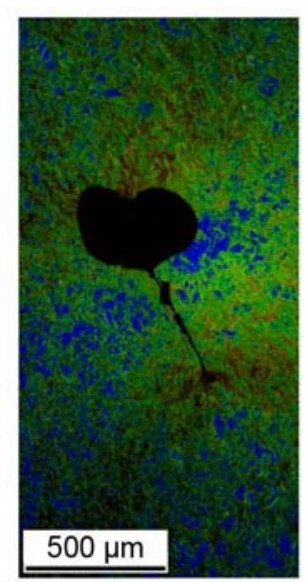

(b)

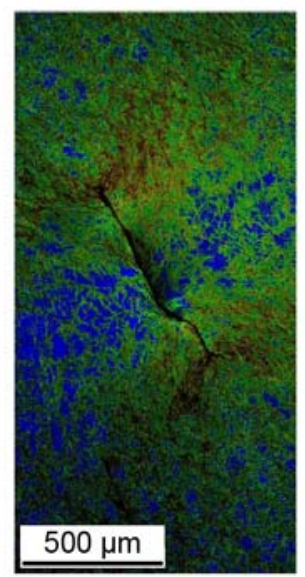

(e)

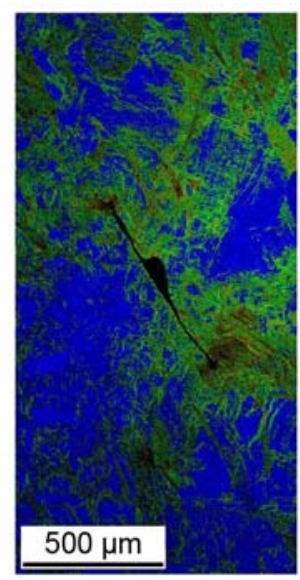

(c)

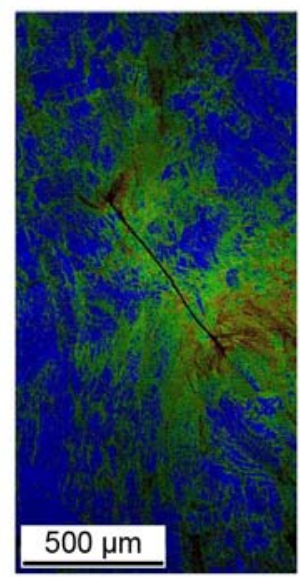

(f)

Figure 15. Kernel average misorientation (KAM) maps computed from EBSD scans obtained after high-strain-rate mechanical testing in the gage area of sample (a) $1 \mathrm{~L}$, (b) $2 \mathrm{~L}$, (c) $3 \mathrm{~L}$, (d) $1 \mathrm{H}$, (e) $2 \mathrm{H}$ and (f) $3 \mathrm{H}$. The left-hand-side inset is a schematic of the location of the zone imaged with respect to the sample.

\section{Conclusion}

In this paper, a comprehensive, in-situ study of plastic localization in dynamically deformed OFHC copper has been conducted using an original experimental protocol that allowed bringing together a number of diagnostics. In particular, the area where localization occurs was characterized at the microstructure scale before, during and after localization. The originality of the method relies especially on the coupled use of a modified SCS and in-situ, ultra-high-speed DIC, which yields access, for the first time, to in-situ, strain measurements at a small scale during a localization process under shear loading.

In the case of our samples, the early stages of plastic localization were actually geometry driven, showing a butterfly localization pattern typical of perforated samples regardless of microstructure or imposed rate of loading. The grain size and strain-rate dependency only became obvious upon further localization. For the two smaller and closest grain sizes, strain-rate dependency of the response was predominant, over a grain size dependency, leading to a narrower and more elongated localization of the highest strains at the local scale and to a concurrent harder macroscopic response at higher strain rate. In these samples, mild lattice rotation and texture formation occurred and was associated with dynamic recrystallization leading to smaller grains in the strain localization area. For a significantly larger grain-size sample, the macroscopic response was nearly identical at both strain rates but largely softer than for the smaller grain sizes. This translated into a wider 
localization pattern in the strain fields compensating for lower values of maximum local strain. The residual lattice rotation was significantly more pronounced for this larger grain size and accompanied with a marked texture formation. In these samples, an interesting and rarely described phenomenon of grain growth-or collective reorientation-accompanied with very low residual KAM was also observed, suggesting a concurrent thermally-assisted relaxation process.

The introduced experimental protocol thus offers the possibility to study in-situ dynamic plastic localization in which time-dependent phenomena such as thermal aspects and dislocation movements play an important role and would be overlooked in a quasi-static approach. Hence a natural perspective of this work would now be to investigate the thermal quantities linked to the localization processes so as to get a better understanding of the energetics involved in dynamic recrystallization and apparent grain growth observed in large-grain samples. This protocol also has the potential of providing useful benchmark experimental data that was not available so far for validating multiscale or crystal plasticity models developed for dynamic applications.

\section{Acknowledgements}

This paper is based upon work supported by the Department of Energy National Nuclear Security Administration under Award Number DE-FC52-08NA28613, which is gratefully acknowledged. The authors thank Brad St. John, Joe Haggerty and Ali Kiani from GALCIT for machining the SCS samples, as well as Mike Lopez from MST-8 Division in LANL for his technical advice. LB and CT also acknowledge the support of Todd Rumbaugh from Highland Imaging for providing the Shimadzu camera.

\section{Bibliography}

Alkhader, M., Bodelot, L., 2012. Large strain mechanical behavior of HSLA-100 steel over a wide range of strain rates. J. Eng. Mater.-T. ASME 134, 1-9.

Bai, Y., Xue, Q., Xu, Y., Shen, L., 1994. Characteristics and microstructure in the evolution of shear localization in Ti-6A1-4V alloy. Mech. Mater. 17, 155-164.

Baig, M., Khan, A. S., Choi, S. H., Jeong, A., 2013. Shear and multiaxial responses of oxygen free high conductivity (OFHC) copper over wide range of strain-rates and temperatures and constitutive modeling. Int. J. Plasticity 40, 65-80.

Bancroft, D., 1941. The velocity of longitudinal waves in cylindrical bars. Phys. Rev. 59, 588-593.

Bodelot, L., Ravichandran, G., 2014. Experimental determination of a representative texture and insight into the range of significant neighboring grain interactions via orientation and misorientation statistics. Int. J. Mater. Res. 105, 117-129.

Bronkhorst, C. A., Cerreta, E. K., Xue, Q., Maudlin, P. J., Mason, T. A., Gray III, G. T., 2006. An experimental and numerical study of the localization behavior of tantalum and stainless steel. Int. J. Plasticity 22, 1304-1335.

Cerreta, E. K., Frank, I. J., Gray III, G. T., Trujillo, C. P., Korzekwa, D. A., Dougherty, L. M., 2009. The influence of microstructure on the mechanical response of copper in shear. Mat. Sci. Eng. A-Struct. 501, 207-219.

Cerreta, E. K., Bingert, J. F., Gray III, G. T., Trujillo, C. P., Lopez, M. F., Bronkhorst, C. A., Hansen, B. L., 2013. Microstructural examination of quasi-static and dynamic shear in high-purity iron. Int. J. Plasticity 40, 23-38. 
Chen, Y. J., Meyers, M. A., Nesterenko, V. F., 1999. Spontaneous and forced shear localization in high-strain-rate deformation of tantalum. Mat. Sci. Eng. A-Struct. 268, 70-82.

Chu, T. C., Ranson, W. F., Sutton, M. A., 1985. Applications of digital-image-correlation techniques to experimental mechanics. Exp. Mech. 25, 232-244.

De Vaucorbeil, A., Bodelot, L., Ravichandran, G., 2009. Modification of the shear compression specimen geometry for high-speed shear compression studies on a high-strength aluminum. California Institute of Technology, Internal Communication.

Dodd, B., Bai, Y., 1985. Width of adiabatic shear bands. Mater. Sci. Tech. 1, 38-40.

Doherty, R. D., 1985. Nucleation and growth kinetics of different recrystallization texture components. Scripta Metall. 19, 927-930.

Doherty, R. D., Chen, L. C., Samajdar, I., 1998. Cube recrystallization texture-experimental results and modeling. Mat. Sci. Eng. A-Struct. 257, 18-36.

Dorogoy, A., Rittel, D., 2005. Numerical validation of the shear compression specimen. Part I: Quasi-static large strain testing. Exp. Mech. 45, 167-177.

Dorogoy, A., Rittel, D., 2005. Numerical validation of the shear compression specimen. Part II: Dynamic large strain testing. Exp. Mech. 45, 178-185.

Dougherty, L. M., Cerreta, E. K., Gray III, G. T., Trujillo, C. P., Lopez, M. F., Vecchio, K. S., Kusinski, G. J., 2009. Mechanical behavior and microstructural development of low-carbon steel and microcomposite steel reinforcement bars deformed under quasi-static and dynamic shear loading. Metall. Mater. Trans. A 40, 1835-1850.

Dougherty, L. M., Cerreta, E. K., Pfeif, E. A., Trujillo, C. P., Gray III, G. T., 2007. The impact of peak shock stress on the microstructure and shear behavior of 1018 steel. Acta Mater. 55, 63566364.

Duffy, J., Chi, Y. C., 1992. On the measurement of local strain and temperature during the formation of adiabatic shear bands. Mat. Sci. Eng. A-Struct. 157, 195-210.

Efstathiou, C., Sehitoglu, H., Lambros, J., 2010. Multiscale strain measurements of plastically deforming polycrystalline titanium: Role of deformation heterogeneities. Int. J. Plasticity 26, 93106.

Escobedo, J. P., Dennis-Koller, D., Cerreta, E. K., Patterson, B. M., Bronkhorst, C. A., Hansen, B. L., Tonks, D., Lebensohn, R. A., 2011. Effects of grain size and boundary structure on the dynamic tensile response of copper. J. Appl. Phys. 110, 033513.

Gao, C. Y., Zhang, L. C., 2012. Constitutive modelling of plasticity of fcc metals under extremely high strain rates. Int. J. Plasticity 32-33, 121-133.

Gray III, G. T., Chen, S. R., Vecchio, K. S., 1999. Influence of grain size on the constitutive response and substructure evolution of MONEL 400. Metall. Mater. Trans. A 30, 1235-1247.

Hall, E. O., 1951. The deformation and ageing of mild steel: III Discussion of results. P. Phys. Soc. B 64, 747-753.

Khan, A. S., Baig, M., Choi, S. H., Yang, H. S., Sun, X., 2012. Quasi-static and dynamic responses of advanced high strength steels: Experiments and modeling. Int. J. Plasticity 30-31, 1-17.

Kolsky, H., 1949. An investigation of the mechanical properties of materials at very high rates of loading. P. Phys. Soc. B 11, 676-700.

Kondo, Y., Maruno, H., Tominaga, H., Soya, H., Etoh, T. G., 2003. An ultrahigh-speed video camera and its applications. 25th International Congress on High-Speed Photography and Photonics 
Li, D. H., Yang, Y., Xu, T., Zheng, H. G., Zhu, Q. S., Zhang, Q. M., 2010. Observation of the microstructure in the adiabatic shear band of 7075 aluminum alloy. Mat. Sci. Eng. A-Struct. 527, 3529-3535.

Marchand, A., Duffy, J., 1988. An experimental study of the formation process of adiabtic shear bands in a structural steel. J. Mech. Phys. Solids 36, 251-283.

Meyers, M., Andrade, U., Chokshi, A., 1995. The effect of grain size on the high-strain, high-strainrate behavior of copper. Metall. Mater. Trans. A 26, 2881-2893.

Meyers, M. A., Ashworth, E., 1982. A model for the effect of grain size on the yield stress of metals. Philos. Mag. A 46, 737-759.

Meyers, M. A., Y.B., X., Xue, Q., Pérez-Prado, M. T., McNelley, T. R., 2003. Microstructural evolution in adiabatic shear localization in stainless steel. Acta Mater. 51, 1307-1325.

Nesterenko, V. F., Meyers, M. A., Wright, T. W., 1998. Self-organization in the initiation of adiabatic shear bands. Acta Mater. 46, 327-340.

Pearson, K., 1900. On the criterion that a given system of deviations from the probable in the case of a correlated system of variables is such that it can be reasonably supposed to have arisen from random sampling. Philosophical Magazine Series 5 50, 157-175.

Pierron, F., Forquin, P., 2012. Ultra-high-speed full-field deformation measurements on concrete spalling specimens and stiffness identification with the virtual fields method. Strain 48, 388-405.

Pierron, F., Sutton, M. A., Tiwari, V., 2011. Ultra high speed DIC and virtual fields method analysis of a three point bending impact test on an aluminium bar. Exp. Mech. 51, 537-563.

Ridha, A. A., Hutchinson, W. B., 1982. Recrystallisation mechanisms and the origin of cube texture in copper. Acta Metall. 30, 1929-1939.

Rittel, D., Lee, S., Ravichandran, G., 2002. A shear-compression specimen for large strain testing. Exp. Mech. 42, 58-64.

Rittel, D., Ravichandran, G., Lee, S., 2002. Large strain constitutive behavior of OFHC copper over a wide range of strain rates using the shear compression specimen. Mech. Mater. 34, 627-642.

Rittel, D., Ravichandran, G., Venkert, A., 2006. The mechanical response of pure iron at high strain rates under dominant shear. Mat. Sci. Eng. A-Struct. 432, 191-201.

Sun, X., Choi, K. S., Liu, W. N., Khaleel, M. A., 2009. Predicting failure modes and ductility of dual phase steels using plastic strain localization. Int. J. Plasticity 25, 1888-1909.

Sutton, M. A., Orteu J. J., Schreier H. W., 2009. Image correlation for shape, motion and deformation measurements. Basic concepts, theory and applications, Springer, New York.

Timoshenko, S., Woinowsky-Krieger, S., 1959. Theory of plates and shells, McGraw-Hill, New York.

Tschopp, M. A., Bartha, B. B., Porter, W. J., Murray, P. T., Fairchild, S. B., 2009. Microstructuredependent local strain behavior in polycrystals through in-situ scanning electron microscope tensile experiments. Metall. Mater. Trans. A 40, 2363-2368.

Wenk, H. R., Tomé, C. N., 1999. Modeling dynamic recrystallization of olivine aggregates deformed in simple shear. J. Geophys. Res-Sol. Ea. 104, 25513-25527.

Wilson, E. B., Hilferty, M. M., 1931. The distribution of chi-square. P. Natl. Acad. Sci. USA 17, 684-688. 
Xue, Q., Gray, G., 2006. Development of adiabatic shear bands in annealed 316L stainless steel: Part I. Correlation between evolving microstructure and mechanical behavior. Metall. Mater. Trans. A 37, 2435-2446.

Zener, C., Hollomon, J. H., 1944. Effect of strain rate upon plastic flow of steel. J. Appl. Phys. 15, 22-32.

Zeng, W., Larsen, J. M., Liu, G. R., 2015. Smoothing technique based crystal plasticity finite element modeling of crystalline materials. Int. J. Plasticity 65, 250-268.

Zhao, Z., Ramesh, M., Raabe, D., Cuitiño, A. M., Radovitzky, R., 2008. Investigation of threedimensional aspects of grain-scale plastic surface deformation of an aluminum oligocrystal. Int. J. Plasticity 24, 2278-2297. 